Aus der Klinik für Klinische Neurophysiologie

(Prof. Dr. med. W. Paulus)

der Medizinischen Fakultät der Universität Göttingen

\title{
Sprechmotorische Planung bei stotternden Erwachsenen und flüssig sprechenden Kontrollpersonen
}

\author{
INAUGURAL-DISSERTATION \\ zur Erlangung des Doktorgrades \\ der Medizinischen Fakultät der \\ Georg-August-Universität zu Göttingen
}

vorgelegt von

Thi Ngoc Linh Hoang

aus

Ho Chi Minh City/Vietnam

Göttingen 2018 


\section{Prüfungskommission}

Betreuer

Korreferentin:

Promotor
Prof. Dr. med. M. Sommer

Prof. Dr. rer. nat. Dipl.-Psych. C. Kiese-Himmel

Prof. Dr. med. M. Oppermann

Datum der mündlichen Prüfung: 09.06.2020 


\section{Inhaltsverzeichnis}

Abbildungsverzeichnis ................................................................................. III

Tabellenverzeichnis ....................................................................................... II

Abkürzungsverzeichnis .................................................................................. IV

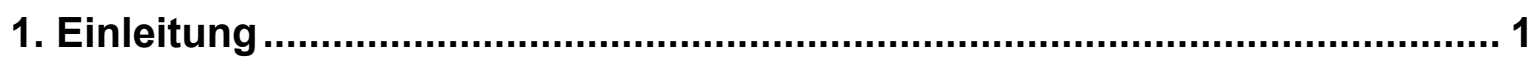

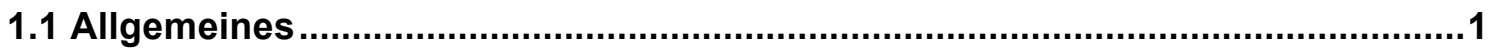

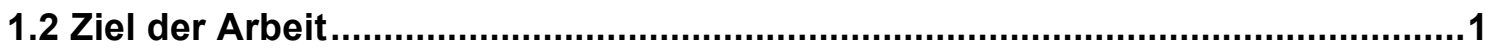

2. Bisheriger Wissensstand der Sprachwissenschaft .................................... 2

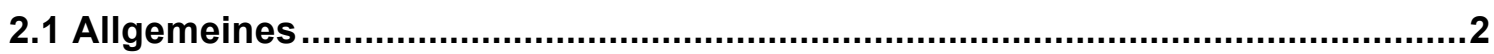

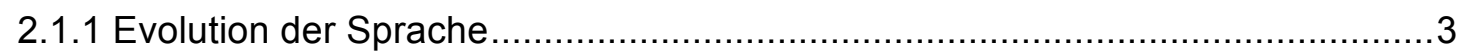

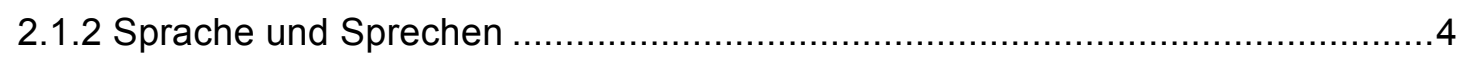

2.1.2.1 Sprache

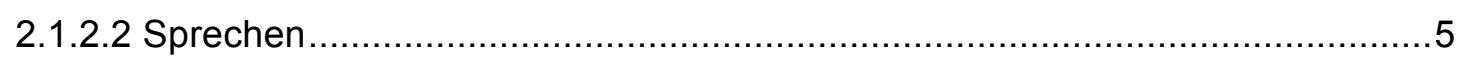

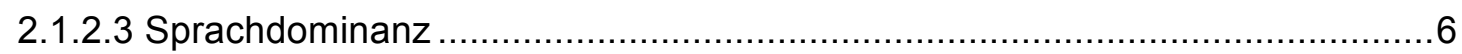

2.2 Anatomie der Sprachzentren im zentralen Nervensystem ...............................

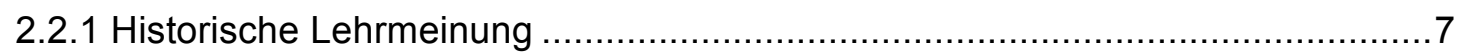

2.2.2 Aktuelle Erkenntnisse zu Modellen der Sprach- und Sprechproduktion .............8

2.3 Neuroanatomische Korrelate der Sprach- und Sprechproduktion .....................9

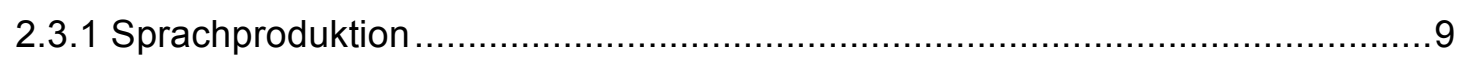

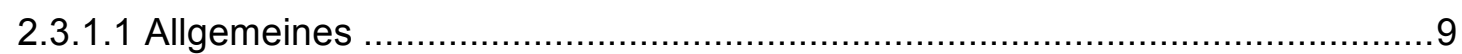

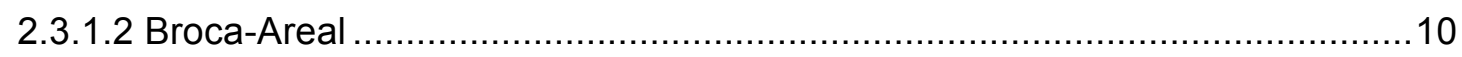

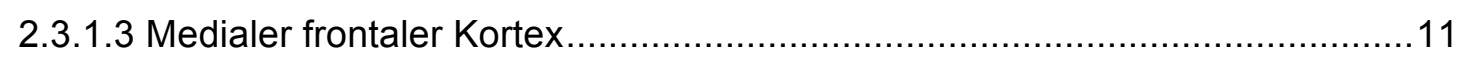

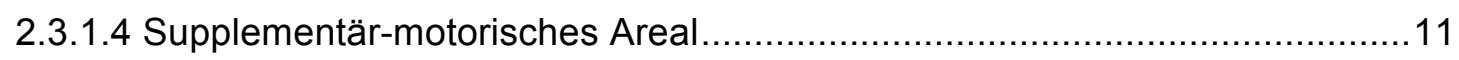

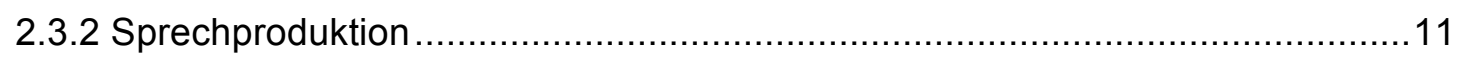

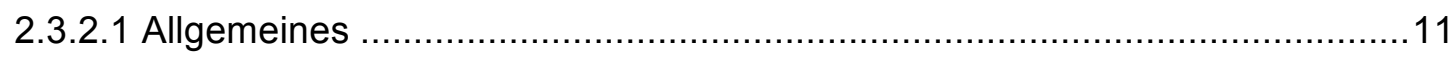

2.3.2.2 Supplementär-motorische-Areal-Basalganglien-Schleife ............................12

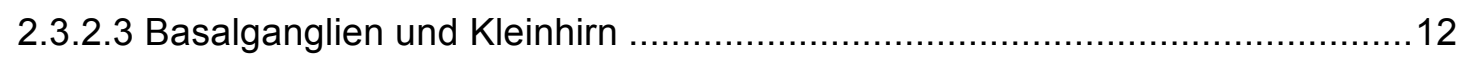

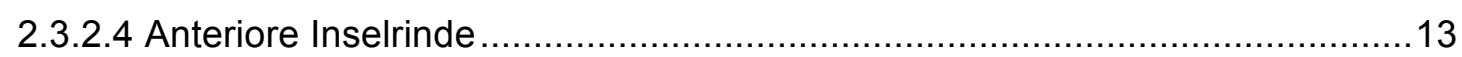

2.3.2.5 Prämotorische Rinde und primär motorische Rinde ..................................13

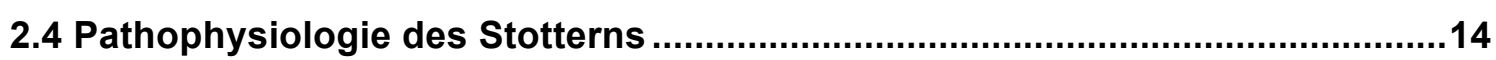

2.4.1 Definition: Chronisch idiopathisches Stottern ................................................. 14

2.4.2 Andere Formen von Sprech-, Stimm- und Sprachstörungen ........................... 14

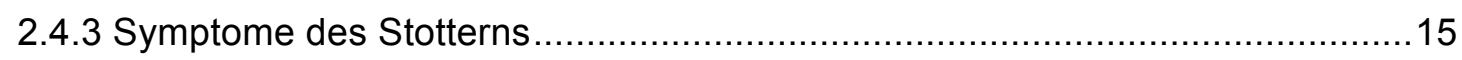

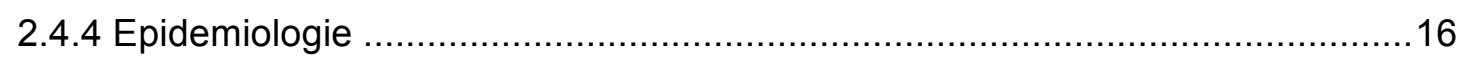

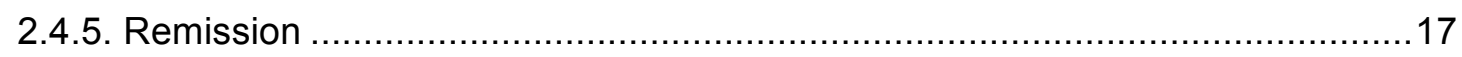

2.4.6 Auswirkungen des Stotterns auf die Lebensqualität ......................................17

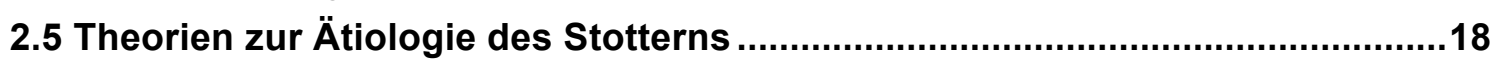

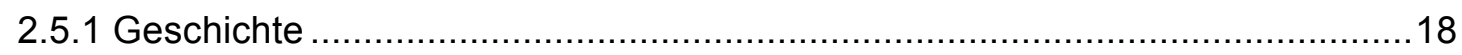

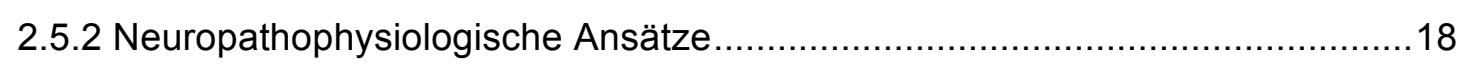

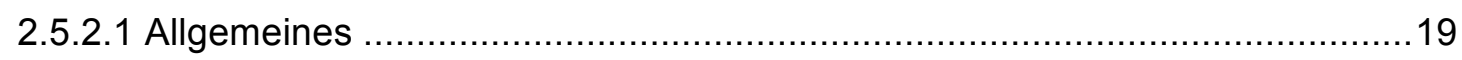


2.5.2.2 Defizite der weißen Substanz in den sprachrelevanten Arealen .................20

2.5.2.3 Theorie der Kompensationsmechanismen ..............................................20

2.5.2.4 Abnormes Bereitschaftspotential ...............................................................20

2.5.2.5 Stottern als Störung des Übergangs beim Sprechen...............................21

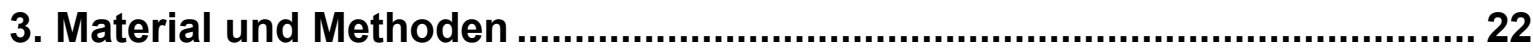

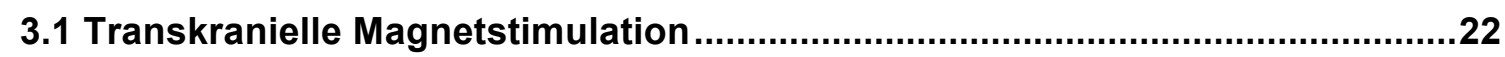

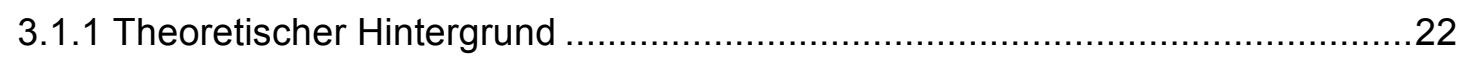

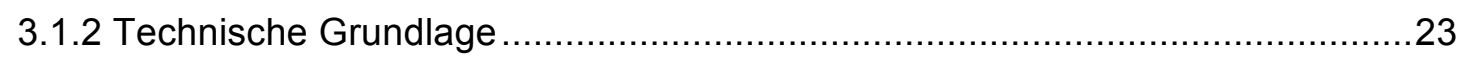

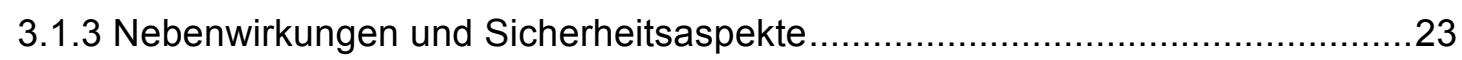

3.1.4 Spulenposition zur Bestimmung der Zungenrepräsentation .........................24

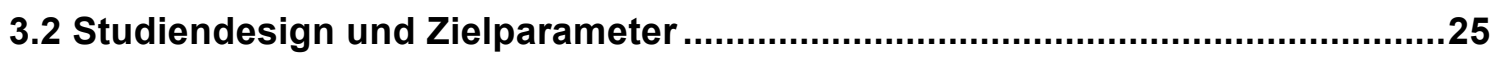

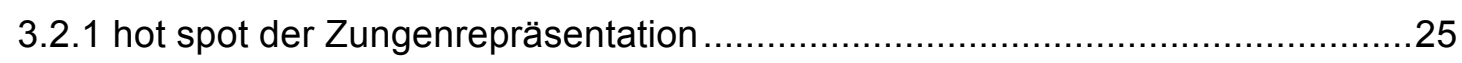

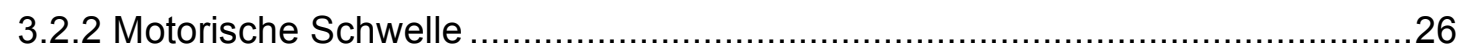

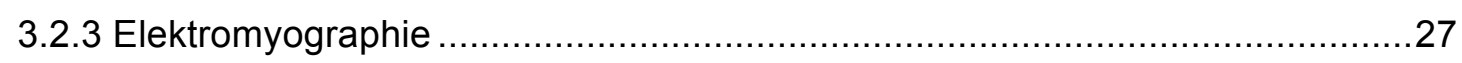

3.2.4 Untersuchungsbedingungen .............................................................. 28

3.2.5 Modulationsfähigkeit der motorisch evozierten Potentiale ...............................28

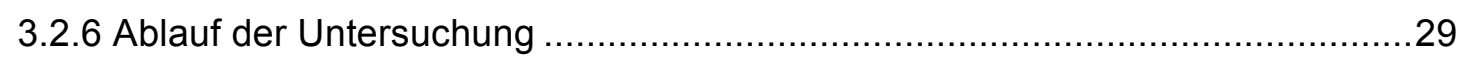

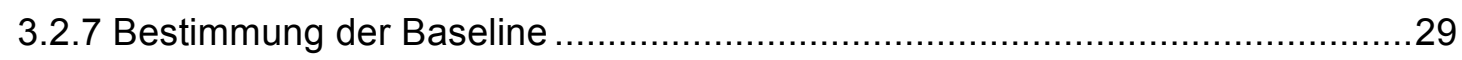

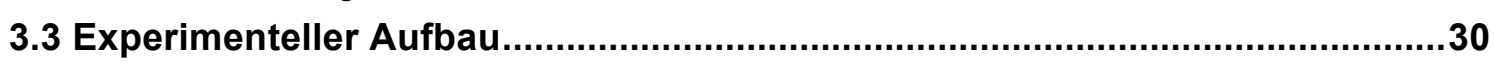

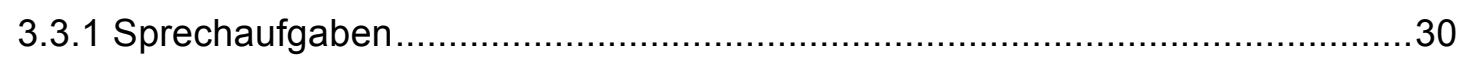

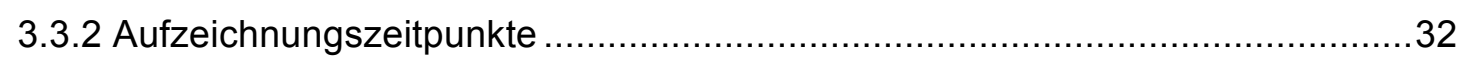

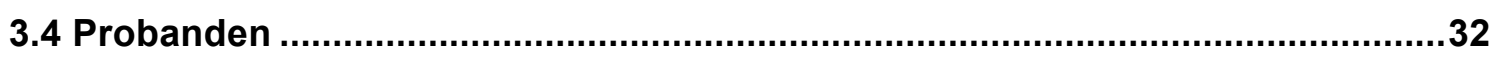

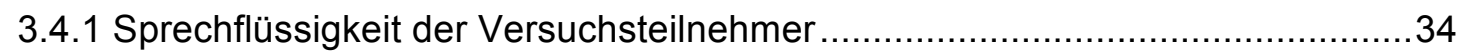

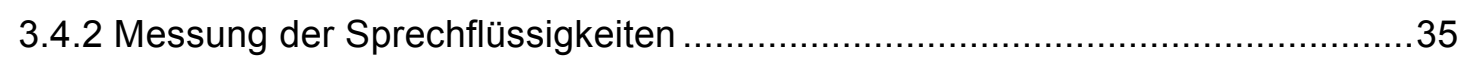

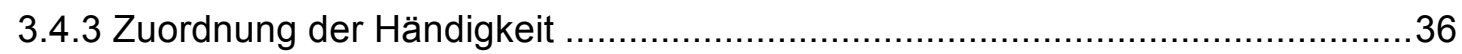

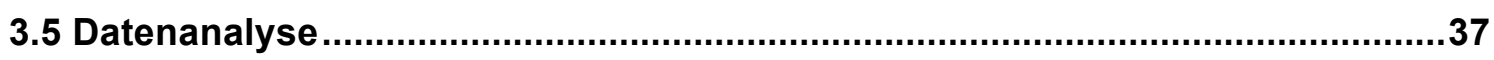

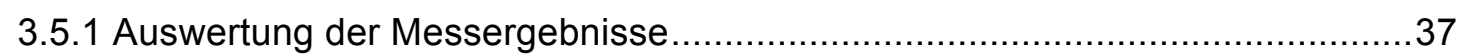

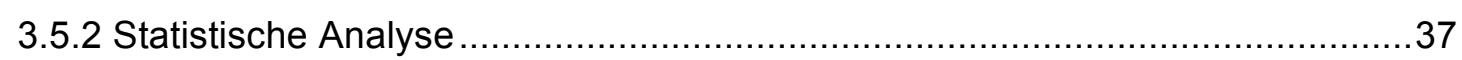

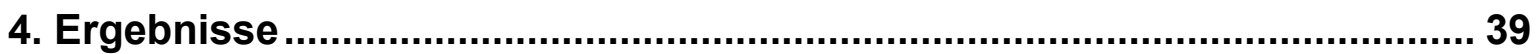

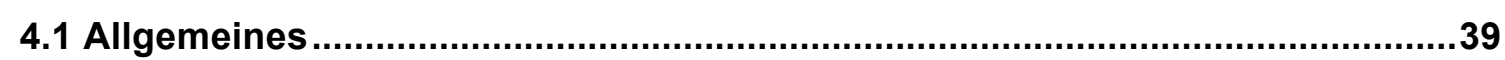

4.2 Neuronale Fazilitation der primär motorischen Zungenrepräsentation während der kontrollierten Prolongation des Reibelauts [f] ..............................................39

4.3 Neuronale Fazilitation der primär motorischen Zungenpräsentation in der

Übergangsphase zum Sprechen des Zielwortes ...................................................40

4.4 Die Stotterschwere korrelierte mit der Erregbarkeitssteigerung des linken sprechmotorischen Kortex ..............................................................................43

4.5 Erregbarkeit der primär motorischen Zungenrepräsentation in Ruhe gemessen als motorische Schwelle.................................................................44

4.6 Erregbarkeit der primär motorischen Zungenrepräsentation in Ruhe

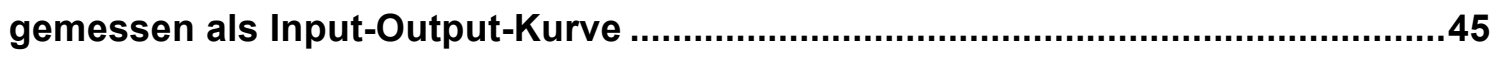

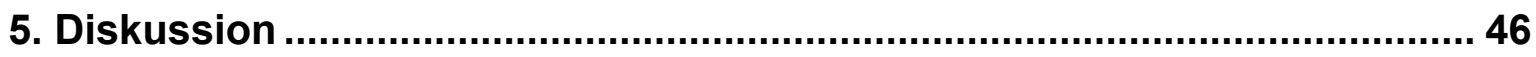

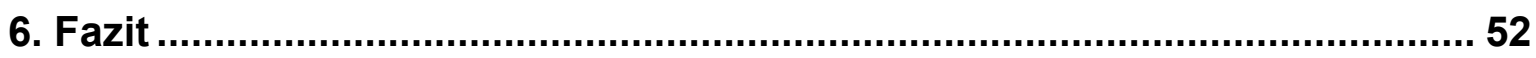




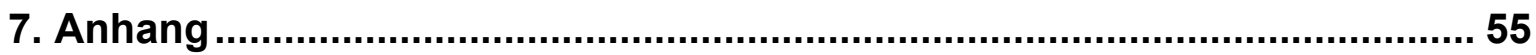

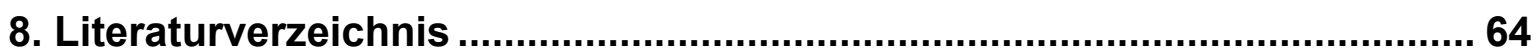

\section{Abbildungsverzeichnis}

Abbildung 1: Vokaltrakt nach Lieberman und McCarthy (2007) ........................ 4 Abbildung 2: Großhirnrinde mit Broca-Areal (Open Textbook Library 2015). ..... 10 Abbildung 3: Versuchsaufbau mit EMG-Ableitungen. …………………….... 27

Abbildung 4: Versuchsablauf während einer Sprechaufgabe. ............................ 31

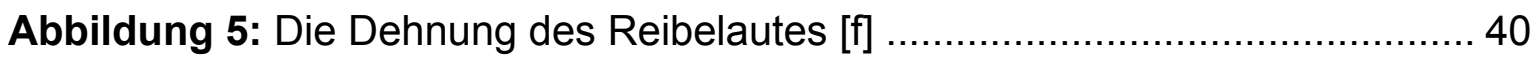

Abbildung 6: Darstellung der Erregbarkeitsprofile während des Sprechens ...... 42

Abbildung 7: Negative Korrelation zwischen Schwergrad des Stotterns und Fazilitation des linken Sprechmotorkortex

Abbildung 8: Input-Output-Kurve für das Erregbarkeitsprofil

des sprechmotorischen Kortex.

\section{Tabellenverzeichnis}

Tabelle 1: Stottersymptomatik nach Natke (2005). ....................................... 15

Tabelle 2: Charakteristische Eigenschaften der Versuchsgruppen..................... 35

Tabelle 3: $p$-Werte der zweiseitig gepaarten $t$-Tests zur Prüfung der sprechinduzierten Erregbarkeitssteigerung.

Tabelle 4: $p$-Werte der zweiseitig ungepaarten $t$-Tests zur Prüfung der Gruppenunterschiede hinsichtlich der Erregbarkeitssteigerung. ... 43 


\section{Abkürzungsverzeichnis}

\%MSO maximaler Stimulator-Output in Prozent

A/D analog/digital

ANOVA Analysis of Variance (Varianzanalyse)

BA Brodmann-Areal

BA44 Brodmann-Areal 44

BA44/BA6 Brodmann-Areal 44/BA6

BA44/B6 Brodmann-Areal 44/B6

BG Basalganglien

CED Cambridge Electronic Design

CIS chronisch idiopatisches Stottern

contra kontralaterale Projektion

EEG Elektroenzephalografie

EMG Elektromyografie

fMRT funktionelle Magnetresonanztomographie

GFI Gyrus frontalis inferior

GODIVA Gradient Order Directions into Velocities of Articulation

ipsi ipsilaterale Projektion

M1 primär motorischer Kortex

MEG Magnetenzephalographie

MEP motorisch evoziertes Potential

MPI Max-Planck-Institut

MRT Magnetresonanztomographie

n. S. nicht significant

PAC primär auditorischer Kortex

PET Positronen-Emissions-Tomographie

PMC prämotorischer Kotex

prä-SMA prä-supplementär motorisches Areal

RMS Ruhemotorschwelle

SD Standardabweichung

SMA supplementär motorisches Areal

SSI Stuttering Severity Instrument

TMS transkranielle Magnetstimulation

UTBAS Unhelpful Thoughts and Beliefs about Stuttering 


\section{Einleitung}

\subsection{Allgemeines}

Studien mit der transkraniellen Magnetstimulation (TMS) zur Untersuchung des primär motorischen Kortex (M1) für orofaziale Strukturen sind in der Literatur kaum zu finden und stellen eine Herausforderung dar. In einer vorangehenden Studie aus dem Labor der Klinik für Klinische Neurophysiologie der Universitätsmedizin Göttingen konnte gezeigt werden, dass die Modulation der Erregbarkeit des M1 während des Ruhezustandes eine reduzierte Dynamik bei stotternden Erwachsenen aufweist (Neef et al. 2011). Diese Arbeit ist die erste onlineBewertung, welche die Erregung der Zungenrepräsentation im M1 während der Sprechaufgaben zeigt.

\subsection{Ziel der Arbeit}

Das Hauptaugenmerk dieser Arbeit liegt in der Erregbarkeit der Zungenrepräsentation im M1. Die vorangehende Zusammenfassung der Literatur hat gezeigt, dass das sprechrelevante Netzwerk bei stotternden Personen Irregularitäten aufweist. Flüssiges Sprechen erfordert ein dynamisches Zusammenspiel von vielen Hirnarealen in höchster zeitlicher und räumlicher Auflösung. Der M1 stellt eine wichtige zentralnervöse Station dar, an welcher ankommende neuronale Signale aus verschiedenen kortikalen und subkortikalen Arealen orchestriert werden, um die schnelle Abfolge der Sprechbewegungen von Kiefer, Lippen, Zunge, Gaumensegel, Kehlkopf und Atemmuskulatur zu steuern. Bekannte neuronale Auffälligkeiten, auch auf der Ebene des M1, stammen aus Untersuchungen, die entweder eine hohe zeitliche Auflösung - mittels der Elektroenzephalografie (EEG) und der Magnetenzephalographie (MEG) oder eine hohe räumliche Auflösung in der Bildgebung wie Positronen-EmissionsTomographie (PET) und Magnetresonanztomographie (MRT) - bieten. Die TMS bietet die Möglichkeit neuronale Prozesse während des Sprechens in einer hohen zeitlichen und räumlichen Auflösung zu monitoren (Classen et al. 1998; Sommer et al. 2001). Es ist anzunehmen, dass es durch die Dysfunktion der 
sprechrelevanten Areale, insbesondere des linken Gyrus frontalis inferior (GFI), zu einer fehlenden Erregbarkeit des linken motorischen Areals bei der Sprechproduktion bei stotternden Personen kommt. Aus diesem Grund stand die Untersuchung der Modulation der neuronalen Erregbarkeit während des Sprechens im Fokus dieser Arbeit. Mit der vorliegenden Arbeit sollten folgende Fragen geklärt werden:

1. Wie ändert sich die Erregbarkeit in der Übergangsphase zwischen Sprechplanung und Sprechausführung in der linken und in der rechten Hemisphäre?

2. Wie ändert sich die Erregbarkeit während der Dehnung eines Sprechlautes in der linken und in der rechten Hemisphäre?

3. Wie unterscheiden sich die Muster der Erregbarkeitsänderungen zwischen Personen, die stottern im Gegensatz zu flüssigen Sprechern?

\section{Bisheriger Wissensstand der Sprachwissenschaft}

\subsection{Allgemeines}

Stottern wird als eine Störung des Sprechens definiert. Jedoch wird Stottern in der Sprachheilpädagogik zusammen mit anderen Redeflussstörungen wie Poltern, Mutismus und Logophobie therapiert (Grohnfeldt 1992), so dass Stottern von einer Sprechstörung nicht klar abzugrenzen ist. Stottern enthält Aspekte sowohl einer Sprech- als auch einer Redeflussstörung. Die Ursache ist bislang unbekannt.

Die psychologischen und sozioökonomischen Auswirkungen des Stotterns sind groß und haben in den letzten Jahrzehnten zahlreiche Untersuchungen zur Entstehung und zur Pathophysiologie sowie zu Behandlungsstrategien des Stotterns veranlasst (Davis et al. 2002; Blumgart et al. 2010).

Die folgenden Abschnitte geben einen Überblick über die Evolution der Sprache sowie die Differenzierung zwischen Sprache und Sprechen. Neurophysiologisch stellt das Stottern eine sprechmotorische Störung dar, die das flüssige Sprechen im Alltag erschwert. Eine detaillierte Definition des Stotterns findet sich in den 
Kapiteln Pathophysiologie und Theorien zur Ätiologie des Stotterns (siehe Punkte 2.4 und 2.5).

\subsubsection{Evolution der Sprache}

Der Zeitpunkt, wann Kommunikation mit verbaler Sprache begonnen hat, kann nicht genau nachvollzogen werden. Historisch gesichert ist nur die Schriftsprache, die auf ca. 3000 v.Chr. datiert worden ist (Jürgens 2006). Nach heutiger Auffassung musste der Sprachevolution eine anatomische und physiologische Entwicklung des Menschen vorangehen, damit eine Lautsprache erst möglich wurde. Es wird beispielsweise angenommen, dass die Zunge im Laufe der Entwicklung in den Rachenraum wanderte, welcher als Resonanzraum dient (Lieberman und McCarthy 2007). Auch die Qualität der auditiven Wahrnehmung (sequentiell und simultan) nahm im Laufe der Evolution zu. Demnach besteht der menschliche Vokaltrakt aus zwei Anteilen, welche in ihrer Ausdehnung etwa gleich groß sind und im rechten Winkel zueinanderstehen. Den horizontalen Teil des Vokaltraktes stellt der Mundraum dar, beginnend an der Mundöffnung bis zum Oropharynx. Den vertikalen Teil des Vokaltraktes macht der Rachenraum aus, der hinter der Zunge beginnt und sich bis oberhalb des Kehlkopfes erstreckt (siehe Abbildung 1). Daneben trugen die neuronalen und kognitiven Entwicklungen des menschlichen Gehirns wesentlich zur Evolution der Sprache bei. Die Relevanz der kognitiven Fähigkeit für die Sprachentwicklung wurde von Fitch und Kollegen (2010) in ihrem Review hervorgehoben. Nach ihrer Auffassung stellt die direkte kortikale Verbindung zur willkürlichen Steuerung des Larynx die Voraussetzung für die Sprachproduktion dar, die bei uns vorzufinden, jedoch bei Schimpansen nicht vorhanden ist (Fitch et al. 2010). Die Entwicklung zum funktionstüchtigen menschlichen Vokaltrakt vollzieht sich während unserer Lebenszeit. Beim Säugling ist die Zunge so positioniert, dass sie während des Saugaktes die Luftzufuhr über die Nase nicht verhindert. Im Alter von 6 bis 8 Jahren ist der menschliche Vokaltrakt, mit welchem wir die Vokale "i”, "u" und "a" aussprechen können, vollständig ausgebildet.

Die anatomischen und neuronalen Entwicklungen, die für den gegenwärtigen Zustand der menschlichen Sprach-, Sprech- und Denkfähigkeiten zuständig sind, 
konnten bereits in Fossilien der Altsteinzeit - 50.000-100.000 Jahre vor unserer Zeit - gefunden werden (Klinke et al. 2005; Jürgens 2006; Lieberman und McCarthy 2007).

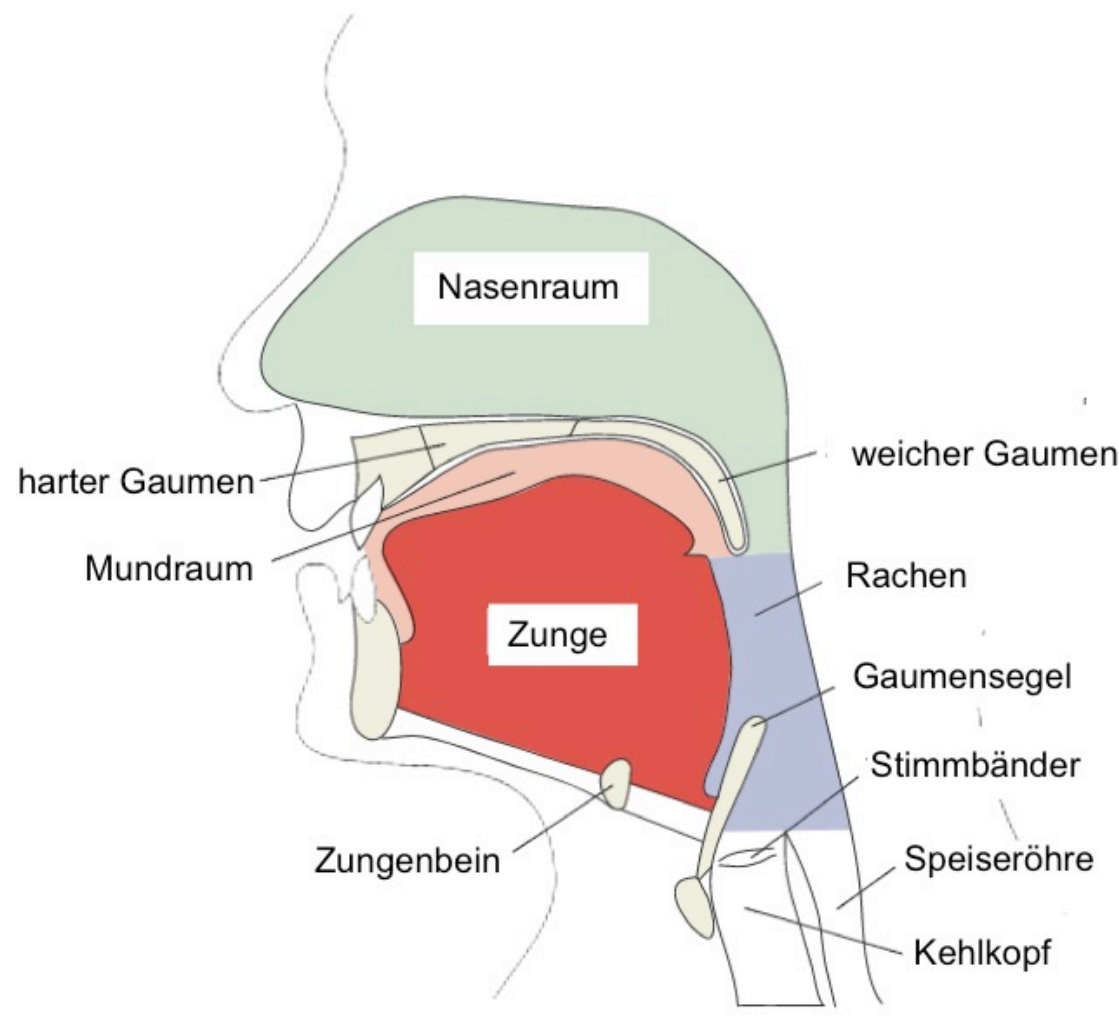

Abbildung 1: Vokaltrakt nach Lieberman und McCarthy (2007).

\subsubsection{Sprache und Sprechen}

\subsubsection{Sprache}

Die vorliegende Arbeit beschäftigt sich mit der Neurobiologie des Sprechens als Grundlage für ein besseres Verständnis der neurophysiologischen Vorgänge beim flüssigen und gestotterten Sprechen. In diesem Zusammenhang ist es wichtig, die neuronalen Funktionen der Sprechproduktion von jenen der Sprachproduktion zu unterscheiden. Zur besseren Abgrenzung dieser beiden Prozesse soll an dieser Stelle kurz auf das Konzept "Sprache“ eingegangen werden. 
Die Sprache wird durch den Inhalt, der durch Bedeutung und Semantik der Sprache gekennzeichnet wird, von der Form unterschieden. Die Form der Sprache wird durch das Gesprochene, durch die Mimik und Gestik, aber auch durch die Schrift charakterisiert. Die Sprache stellt ein Symbolsystem dar, welches als Medium zur Kommunikation dient.

Klinisch ist eine Aphasie, bei welcher die Sprachstörung durch Beeinträchtigung des Sprachverständnisses und/oder der Wort- und Satzsemantik gekennzeichnet ist, von der Dysarthrie zu unterscheiden. Bei dieser liegt eine Sprechstörung vor, die durch verwaschene Sprache und undeutliche Artikulation charakterisiert wird (Primaßin et al. 2017). Stottern ist eine Redeflussstörung, die je nach Lehrmeinung unterschiedlich zugeordnet wird. Während Büchel und Sommer (2004) Stottern als Störung motorischer Genese betrachten, vermuten andere Wissenschaftler auch eine Störung der Sprachkompetenz, beispielsweise der Grammatikkompetenzen (Weber-Fox und Usler 2015).

Bei Erwachsenen kommt Stottern bei einem Wortanfang mit einem Konsonanten vor. Im Gegensatz dazu werden bei Kindern die Inhaltswörter mehr als bei Funktionswörtern gestottert. Bei langen Wörtern oder zusammenhängender Sprache wird mehr gestottert als bei einfacher und nicht zusammenhängender Sprache. Die ersten Wörter im Satz und die Anfangslaute sowie betonte Silben werden oft gestottert (Natke 2005).

Die Sprache beschränkt sich nicht nur auf das Gesprochene, sondern kann darüber hinaus Elemente wie Gestik und Mimik enthalten, die den Inhalt einer Mitteilungsbotschaft wiedergeben. Ursprünglich entwickelte sich die Wortsprache aus der Körpersprache, was sowohl menschheits- als auch individualgeschichtlich nachzuvollziehen ist. Die verbale Sprache ist das höchstentwickelte und zuletzt erworbene Verständigungssystem (Lurija 1982; Hickok und Poeppel 2004).

\subsubsection{Sprechen}

Das Sprechen stellt eine der kompliziertesten motorischen Fertigkeiten dar, die der Mensch erlernt und ausführt. Am flüssigen Sprechen sind zahlreiche Muskeln und die drei Funktionsbereiche Atmung, Phonation und Artikulation beteiligt (Natke 
2005). Das Ergebnis des koordinierten Sprechvorgangs ist das Lautwerden der Sprache. Das Sprechen an sich erfordert vielseitige kognitive Prozesse, mit denen eine verbale Kommunikation erst möglich wird. Die kognitiven Fähigkeiten für die Sprechproduktion scheinen bei den Schimpansen, die uns genetisch am nächsten verwandt sind, zu fehlen. Trotz intensiven Trainings vom Kleinkindalter an haben die Schimpansen nicht die Fähigkeit, die menschliche Sprache nachzuahmen (Hayes 1951).

In der vorliegenden Arbeit wurde der Vorgang des Sprechens auf zerebraler Ebene untersucht. Als Hypothese wird angenommen, dass Stottern eine neuronale Übergangsstörung darstellt.

\subsubsection{Sprachdominanz}

Die Sprachzentren sind bei bis zu 90\% der Menschen auf der linken Hemisphäre des Gehirns angelegt. Warum das so ist, lässt sich nicht ergründen. Bei lediglich $10 \%$ aller Menschen liegen die Sprachzentren sowohl auf der linken als auch auf der rechten Hemisphäre. Es gibt jedoch Untersuchungen, die belegen, dass die rechte Hemisphäre ebenfalls sprachrelevante Hirnareale enthält. Eine erhöhte Aktivität wurde in der rechten anterioren Inselrinde bei stotternden Personen beobachtet (Brown et al. 2005). Die Sprachqualität wird durch die anatomische Anordnung der Sprachzentren auf beiden Hemisphären ohne eine sprachrelevante Hemisphärendominanz nicht zwingend besser. Es scheinen dadurch Sprachstörungen wie Stottern sowie langsamer und unbeholfener Sprachstil aufzutreten. Während die linke Hemisphäre des Gehirns für das Verstehen und die Produktion der gängigen Sprache zuständig ist, ist die rechte Hemisphäre des Gehirns für die prosodische Sprachproduktion verantwortlich (Klinke et al. 2005; Natke 2005). Bei einer frühkindlichen Hirnschädigung der linken Hemisphäre kann die Ausbildung der Sprachzentren auf der rechten Hemisphäre so ausgeprägt sein, dass die rechte Hemisphäre die Sprachfunktion übernimmt (Geschwind und Galaburda 1985; Watkins et al. 2001; Vigneau et al. 2006). Gemäß der Lateralisierungshypothese, welche in den 1920er Jahren von Orton (1927; 1928) und Travis (1978) formuliert wurde, weisen stotternde Personen keine klaren kortikalen Dominanzverhältnisse für Sprechen und Sprache 
auf. Ein zerebraler interhemisphärischer Konflikt der konkurrierenden Hemisphären des Gehirns führe zur Störung des Sprechablaufs (Natke 2005). Weiterführende Studien zur Lateralisierungshypothese konnten allerdings keine eindeutigen Unterschiede in der zerebralen Dominanz von Sprache und Sprechen bei Stotternden und Gesunden nachweisen (Jones 1966; Walle 1971; Andrews et al. 1972). Es sind jedoch in zahlreichen bildgebenden und elektroenzephalographischen Untersuchungen Lateralisierungsanomalien gefunden worden, so dass die Lateralisierungshypothese zwar nicht bestätigt, aber auch nicht widerlegt werden konnte.

\subsection{Anatomie der Sprachzentren im zentralen Nervensystem}

\subsubsection{Historische Lehrmeinung}

Der folgende Absatz fasst die sogenannte „klassische Lehrmeinung“ zusammen, welche nicht dem aktuellen Stand der Forschung entspricht. Im zentralen Nervensystem werden definierte Kortexareale den Sprachzentren zugeordnet. Makroanatomisch sind dies das Broca- und das Wernicke-Areal. Bereits im Jahre 1874 wurde das Wernicke-Areal von dem deutschen Neurologen Carl Wernicke (1848-1905) als das sensorische Sprachzentrum beschrieben. Seine Afferenzen erhält das Areal vom primär auditorischen Kortex (PAC). Seine Efferenzen werden in die kortikalen Assoziationsfelder projiziert, um dort verarbeitet und weitergeleitet zu werden. Das sensorische Sprachzentrum (Wernicke-Areal BA42/22), welches sich an der Konvexität des hinteren Drittels des linken Schläfenlappens und in der Nähe der Hörrinde befindet, dient dem Verstehen von gehörten sowie gelesenen Wörtern, Sätzen und damit der Verarbeitung der Sprache. Das motorische Sprachzentrum (Broca-Areal BA44/45) befindet sich im GFI, unmittelbar vor der Basis des prämotorischen Kortex und in dichter Nachbarschaft zu den unteren Anteilen des präzentralen Gyrus. Es ist für die Steuerung der Sprechwerkzeuge (Kehlkopf, Zunge, Gaumen und Lippen) zuständig. Das Broca-Sprachzentrum wurde erstmals im Jahr 1862 von dem französischen Chirurgen Paul Broca beschrieben (1824-1880). Eine Schädigung des Broca-Areals führt zur motorischen Aphasie, bei der das Sprachverständnis erhalten bleibt, während die Artikulation gestört ist. Von einer Wernicke-Aphasie spricht man, wenn das 
Sprachverständnis geschädigt ist und sinnentstellte Sätze gesprochen werden (Benninghoff und Fleischhauer 1985; Trepel 2005; Schwegler und Lucius 2016).

Eine funktionsfähige Überleitung vom motorischen Sprachzentrum zu den ausführenden Sprechorganen ist für ein flüssiges Sprechen essentiell. Darüber hinaus sorgt das motorische Sprachzentrum für die Füllung der Räume zwischen den sinnhaltigen Wörtern mit sog. Interjektionen (Laute wie „ähhh“ oder „hmm“). Bei der flüssig gesprochenen Sprache schafft das motorische Sprachzentrum elegante und grammatische Überleitungen. Sind die motorischen Sprachzentren beeinträchtigt, kann die Sprache sogenannte Füllwörter und Sprechdenkgeräusche in Form von unbeholfenen "ähs" und "mms" enthalten (Schwegler und Lucius 2016).

2.2.2 Aktuelle Erkenntnisse zu Modellen der Sprach- und Sprechproduktion

Nach den heutigen wissenschaftlichen Erkenntnissen sind, abgesehen von den bereits bekannten Broca- und Wernicke-Arealen, noch andere zerebrale Areale und neuronale Verbindungen verantwortlich, das Sprechen und somit das Lautwerden der Sprache zu ermöglichen. In zahlreichen Arbeiten wurden die funktionellen, strukturellen und anatomischen Aufgaben des Broca- und des Wernicke-Areals mit ihren neuronalen Verbindungen zu anderen Hirnarealen, die zur Produktion der Sprache beitragen, untersucht (Friederici 2002; Hickok und Poeppel 2004; Vigneau et al. 2006; Eickhoff et al. 2009). Dabei interagieren alle sprachrelevanten Hirnareale miteinander, welche Lichteim (1885) als Interaktionen des auditiven Zentrums, des motorischen Zentrums und des konzeptuellen Zentrums bezeichnete.

In der Literatur werden zwei Verbindungen (ventrale und dorsale Bahnen) beschrieben, die in die Sprachproduktion involviert sind. Die Untersuchungen ergaben, dass dorsale Bahnen die audio-motorische Integration „Sprachlaute-zusprechmotorischem Programm = Form" (auditory-motor integration) unterstützen, während ventrale Bahnen die Zuordnung „Sprachlaute-zu-Bedeutung = Inhalt“ 
(sound-to meaning mapping) fördern (Hickok und Poeppel 2004; Catani et al. 2005; Hickok und Poeppel 2007; Rauschecker und Scott 2009; Friederici 2011).

Es ist bekannt, dass der dorsale Pfad I, welcher der audio-motorischen Integration dient, primäre Bedeutung für den frühen Spracherwerb hat, während der dorsale Pfad II sich erst später entwickelt und eine niveauvolle semantische und syntaktische Sprachfunktion formt (Brauer et al. 2011).

Hickok (2012) versuchte, den psycholinguistischen und den Motor-gesteuerten Ansatz (motor control) der Sprachproduktion in dem Sprachmodell hierarchical state feedback control model zu integrieren. Er ist der Meinung, dass Sprache einer hierarchischen Organisation unterliegt. So beginnt die Sprachproduktion auf der linguistischen Ebene, die daraufhin die Sprachebene anregt. Die angekommenen Informationen auf der Sprachebene (word level) werden weiter auf der sensorischen und motorischen Ebene, also der höchsten kortikalen Ebene der Rückkopplungsregelung (feedback control), in die sogenannte HörrindenSulcus-lateralis-BA44-Schleife (auditory-Sylvian fissure-BA44-loop) eingespeist. Diese wiederum projiziert die verarbeitete Information auf die niedrigere Ebene der somatosensorischen Kortex-Kleinhirn-Motorkortex-Schleife (somatosensorycerebellum-motor cortex-loop). Schließlich erfolgt der endgültige Befehl an den Vokaltrakt, der das Sprechen ausführt, unter ständiger Rückkopplungsregelung der akustischen und somatosensorischen Systeme (acoustic feedback, somatosensory feedback).

\subsection{Neuroanatomische Korrelate der Sprach- und Sprechproduktion}

\subsubsection{Sprachproduktion}

\subsubsection{Allgemeines}

Damit Sprachlaute entstehen können, muss die präverbale Botschaft, die die Anforderungen einer bestimmten Sprache erfüllen muss, über eine Reihe von Schritten transformiert werden. In den einzelnen Schritten werden verschiedene Informationen vom Gedächtnis abgerufen (Semantik, Syntaktik und Phonologie) und in einer großen Struktur kombiniert, die als Vereinigung bezeichnet wird 
(Hagoort und Levelt 2009). Anatomisch sind Anteile des Temporallappens, des GFI und ein Teil des Broca-Areals in die Satzbildung involviert (Friederici 2011).

\subsubsection{Broca-Areal}

Das Broca-Areal (siehe Abbildung 2) liegt im GFI, der in drei Anteile, die Pars orbitalis, Pars triangularis und Pars opercularis aufgeteilt ist. Bei Rechtshändern befindet sich das Broca-Areal meist auf der linken Hemisphäre des Gehirns (Benninghoff und Fleischhauer 1985). Zytoarchitektonisch gehören zum BrocaAreal das linke Brodmann-Areal BA44, die Pars operculum und das linke Brodmann-Areal BA45, die Pars triangularis (Trepel 2005).

Das Broca-Areal ist der Eintrittsort aller sprachlichen Informationen und gleichzeitig die Weiterleitungsstelle an die danach folgenden Vernetzungspunkte (Friston et al. 2003). Es ist der Ort der Sprachorganisation und -produktion (Benninghoff und Fleischhauer 1985). In den vier experimentellen Untersuchungsbedingungen (semantisches, phonologisches, syntaktisches und freies Sprechen) wird das Broca-Areal BA44 aktiviert. Es stellt die Endphase der Wortfindung dar, welche die tatsächliche Artikulation initiiert (Eickhoff et al. 2009).

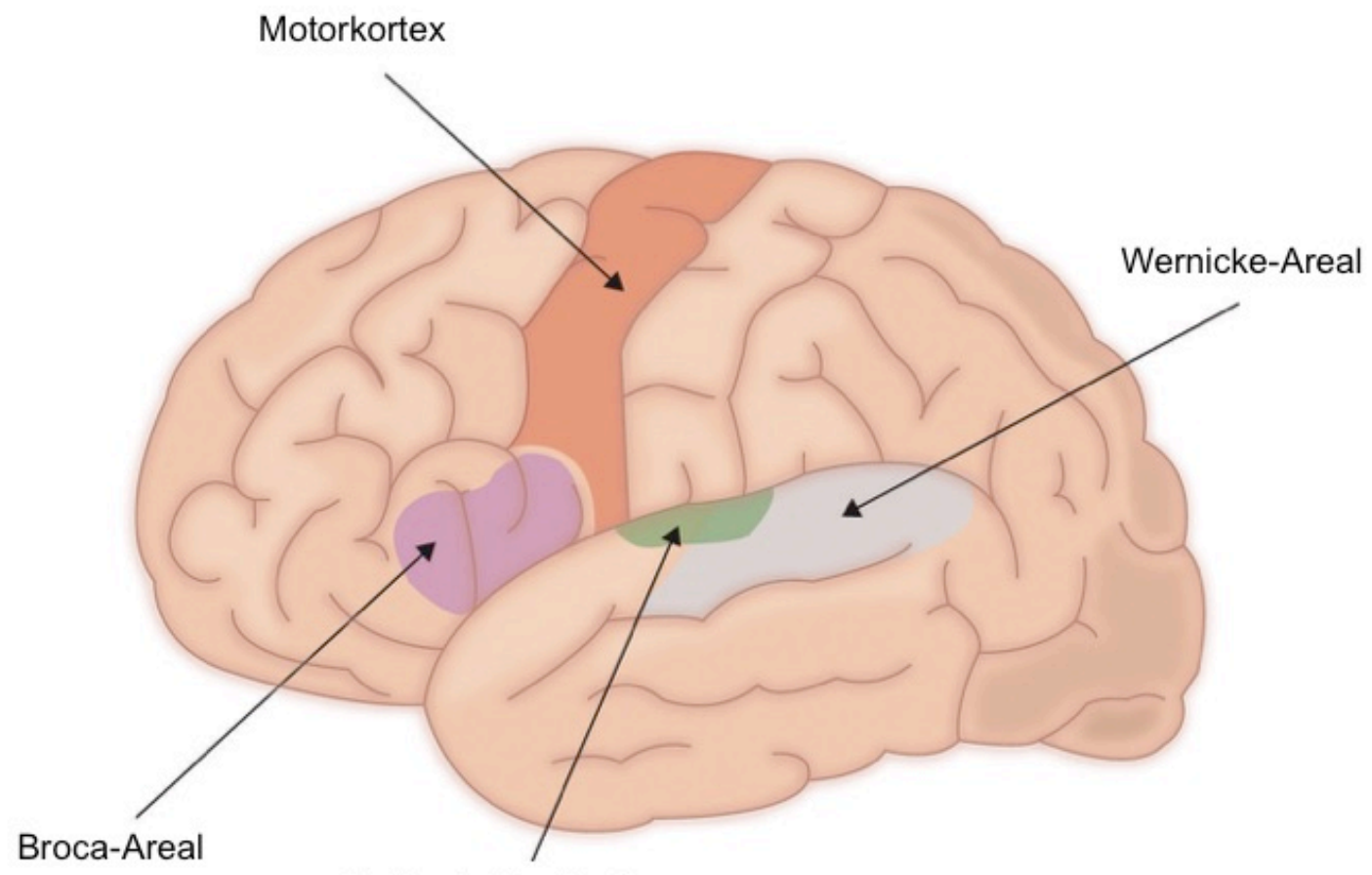

Auditorischer Kortex

Abbildung 2: Großhirnrinde mit Broca-Areal (Open Textbook Library 2015). 


\subsubsection{Medialer frontaler Kortex}

Der mediale frontale Kortex enthält das posteriore supplementär motorische Areal (SMA) und das anteriore prä-SMA, welche bei den Sequenzierungs- und Sprachproduktionsaufgaben einbezogen werden. Während das prä-SMA den strukturellen Entwurf codiert, setzt das SMA den geplanten Sprechakt frei (Bohland et al. 2010). Phonologische Prozesse, insbesondere die Initiation des Motorprogramms während der Sprachproduktion, wurden unter anderem dem linken SMA zugeschrieben (Bohland und Guenther 2006).

\subsubsection{Supplementär-motorisches Areal}

In den letzten Jahren wurde dem SMA in Bezug auf die sogenannte PhonemProduktion (Laute-Produktion) eine größere Bedeutung beigemessen. Es gibt Hinweise darauf, dass die SMA-Basalganglien-Schleife (SMA-BG-Ioop) bei der Initiation des Motorprogramms der phonematischen Wortform involviert ist. Peeva et al. (2010) stellten die Hypothese auf, dass das sprechmotorische Programm von der SMA initiiert wird, während die Basalganglien konkurrierende Bewegungen inhibieren.

\subsubsection{Sprechproduktion}

\subsubsection{Allgemeines}

An der motorischen Sprechproduktion sind u.a. subkortikale Hirnareale beteiligt. In einer bildgebenden Studie mittels fMRT wurde der Prozess der Phonem-Bildung im linken SMA (Pallidum), im posterior-superioren Gyrus und im superior-lateralen Zerebellum nachvollzogen, während der Prozess der Silben-Bildung im linken ventralen Prämotorkortex beobachtet wurde. Der Prozess der Prosodie-Produktion wurde im rechten superior-lateralen Zerebellum gefunden (Peeva et al. 2010). 


\subsubsection{Supplementär-motorische-Areal-Basalganglien-Schleife}

Es gibt Hinweise dafür, dass für die Initiierung des motorischen Sprechprogramms das supplementär motorische Areal zuständig ist, während die Basalganglien unerwünschte Bewegungen inhibieren. Die Informationen erhält der Globus pallidus vom subthalamischen Nukleus, welcher direkt vom motorischen und prämotorischen Areal Informationen empfängt. Zusammenfassend kann man davon ausgehen, dass die Sprechmotorik, zumindest für den Prozess der Phonem-Bildung, durch die sogenannte SMA-Basalganglien-Schleife initiiert wird, bei der die Thalamuskerne als Informationstransfer dienen.

\subsubsection{Basalganglien und Kleinhirn}

Während in den Basalganglien das sprechmotorische-Programm (vocalizationmotor-program) ausgewählt und initiiert wird, vollzieht das Kleinhirn die zeitliche Verfeinerung der verschlüsselten vorbereiteten Sequenzen in eine flüssige Sprechaktion (Eickhoff et al. 2009). Über Schleifen (loops) der Basalganglien werden Informationen von anderen Kortexarealen (z. B. solchen aus dem Operculum frontale) mit den Basalganglien verbunden (Benninghoff und Fleischhauer 1985). Wie Harrington und Haaland (1998) bereits herausgefunden haben, sind die Basalganglien bei der Sequenzierung der motorischen Sprachproduktion involviert. Eine Schädigung des Nucleus caudatus führt zur Perseveration und Paraphasie (Kreisler et al. 2000). Die Interaktion zwischen dem superioren Zerebellum, dem medialen und lateralen Prämotor-Kreislauf sowie dem primär motorischen Kortex dient der Transformation der Phonem- und Silbenbildung und der nachfolgenden Bewegung von Lippen, Kiefer und Zunge sowie von allen artikulatorisch ausführenden Sprechorganen (Peeva et al. 2010). 


\subsubsection{Anteriore Inselrinde}

Die Inselrinde fungiert bei der Sprechproduktion wie ein Schalter, welcher die kognitiven Aspekte der Sprache bei der Vorbereitung auf die tatsächliche Stimmbewegung in den motorischen Schaltungen der Basalganglien und des Kleinhirns umlegt. Eickhoff et al. (2009), Ackermann und Riecker (2004) sowie Guenther (2006) postulierten, dass die anteriore Inselrinde wie ein Filter fungiert, der die hohe Anzahl der beteiligten Muskeln für die Artikulation und Phonation koordiniert.

\subsubsection{Prämotorische Rinde und primär motorische Rinde}

Die prämotorische Rinde (PMC) erhält die Informationen von subkortikalen Strukturen, insbesondere den Basalganglien. Anschließend werden die Informationen in den M1 weitergeleitet (Eickhoff et al. 2009). Die PMC, der das SMA zugeordnet wird, dient als Übersetzer der geplanten Bewegung in ein Muster von spezifischen Muskelkontraktionen (Jueptner und Weiller 1998), welches letztendlich vom M1 ausgeführt wird (Eickhoff et al. 2009). Während die Sprechvorbereitung (preparation) durch die Verbindung zwischen der Inselrinde und den Basalganglien vollzogen wird, wird das Ausführen der Artikulation erst durch die Efferenzen der Basalganglien in dem M1 bewerkstelligt. Eine Schädigung des PMC (z. B. des SMA) und des Broca-Areals, insbesondere des posterioren Anteils des GFI, kann zur Beeinträchtigung des Übergangs von der Sprechplanung zur Sprechausführung führen. Diese Störung kann z. B. bei stotternden Personen auftreten, bei denen eine Störung der Sprachplanung und eine daraus resultierende motorische Sprechstörung anzunehmen ist (Eickhoff et al. 2009). 


\subsection{Pathophysiologie des Stotterns}

\subsubsection{Definition: Chronisch idiopathisches Stottern}

Das chronisch idiopathische Stottern (CIS) ist die häufigste und bekannteste Redeflussstörung unbekannter Pathophysiologie, die sich ohne einen offensichtlichen Grund in der Kindheit entwickelt. Unter Stottern versteht man eine Redeflussstörung, die auch als Balbuties (lat. balbutire: stammeln, stottern, lallen) bezeichnet wird (Natke 2005). Nach der Definition von Wingate (1964) wird die verbale Äußerung bei stotternden Menschen durch unwillkürlich hörbare, lautlose Repetitionen und Prolongationen von Tönen und Silben unterbrochen. Diese Definition wird häufig gebraucht, weil sie Sprechunflüssigkeiten beschreibt ohne die Begleitsymptome des Stotterns hervorzuheben. Stottern beschreibt kein Krankheitsbild, wobei unter dem Begriff "Stottern" sowohl die Störung (disorder) als auch Symptome verstanden werden (Büchel und Sommer 2004).

\subsubsection{Andere Formen von Sprech-, Stimm- und Sprachstörungen}

Vom CIS sind erworbenes Stottern (acquired stuttering), Poltern, Mutismus und spasmodische Dysphonie zu unterscheiden. Erworbenes Stottern tritt meist plötzlich im Erwachsenenalter und durch neuronale Schädigungen (neurogenes Stottern) oder psychische Ursachen (psychogenes Stottern) auf. Neurogenes Stottern wird häufig von Symptomen wie Aphasie, Apraxie und Dysarthrie begleitet (Ringo und Dietrich 1995). Beim Poltern ist die Sprechfrequenz zu schnell. Es kommt zu überstürzten und undeutlichen Formulierungen sowie häufige Repetitionen von Silben oder Wörtern. Beim Mutismus verweigern die Kinder aus unbekannter Ursache die bereits erworbene Sprechfähigkeit. Bei der spasmodischen Dysphonie handelt es sich um eine zentrale Störung, die zur Anspannung der Stimmlippen führt und die Stimmabbrüche oder knarrende Stimmeinsätze erzeugt (Natke 2005). 


\subsubsection{Symptome des Stotterns}

Die Beeinträchtigung durch das CIS wird in äußere und innere Symptome unterschieden. Die äußeren Symptome unterteilen sich nochmals in die Kernsymptomatik wie Laut- und Silbenwiederholungen, Lautprolongationen und Blöcke sowie die sogenannte Sekundärsymptomatik, wie Muskelanspannung, Flucht- und Vermeidungsverhalten. Zu den inneren Symptomen zählen negative Gefühle wie Sprechangst, Scham und ein geringes Selbstwertgefühl, was die Lebensqualität von stotternden Menschen stark mindert (siehe Tabelle 1; Natke 2005).

\begin{tabular}{|c|c|c|c|}
\hline \multirow[t]{8}{*}{ Symptome } & Kernverhalt & $\begin{array}{l}\text { Repetitionen } \\
\text { Prolongation } \\
\text { Blocks }\end{array}$ & $\begin{array}{l}\text { > ke-ke-ke-kann }<< \\
>>\text { ffffast }<< \\
\text { >>----kann }<<\end{array}$ \\
\hline & Sekundärsymptomatik & Muskelspannung & Tremor \\
\hline & & Fluchtverhalten & $\begin{array}{l}\text { Neuansetzen, Vocal Fry, } \\
\text { Mitbewegungen }\end{array}$ \\
\hline & & Vermeidungsverhalten & Vermeidung von \\
\hline & & & Sprechsituationen, \\
\hline & & & Substitutieren von \\
\hline & & & Wörtern, Aufschiebung \\
\hline & & & $\begin{array}{l}\text { durch Flicklaute und - } \\
\text { wörter, Einschübe, Starter }\end{array}$ \\
\hline Innere & & Gefühle & Angst, Frustration, Scham, \\
\hline \multirow[t]{2}{*}{ Symptome } & & & Aggression \\
\hline & & Einstellungen & Negatives Bild \\
\hline
\end{tabular}

Tabelle 1: Stottersymptomatik nach (Natke 2005). 


\subsubsection{Epidemiologie}

Stottern betrifft ca. 5\% der Kinder (vom Kleinkindalter bis zur Pubertät). Dabei tritt die Sprechstörung bei stotternden Kindern bis zu 50\% vor dem 4. Lebensjahr, $75 \%$ vor dem 6. Lebensjahr und $100 \%$ vor dem 12. Lebensjahr auf (Andrews 1985). Ein genauer Zeitpunkt für das Auftreten des Stotterns kann jedoch nicht festgelegt werden. Häufig wird von den Eltern ein einschneidendes Ereignis, wie die Geburt eines Geschwisterteils oder ein Todesfall in der Familie angegeben (Natke 2005). Diese Annahme wird jedoch nicht von vielen Autoren geteilt. So ist Van Riper (1982) der Ansicht, dass ein spezielles Ereignis dem Stottern nicht vorausgeht.

Häufig tritt das Stottern schleichend auf, weshalb fehlerhafte neuronale Entwicklungsprozesse eine mögliche Ursache des Stotterns darstellen können (Yairi 1983; Sandrieser und Schneider 2003). Bei jedoch einem Drittel der Kinder konnte ein abrupter Beginn des Stotterns beobachtet werden, der als neurophysiologische Veränderung zu deuten ist (Yairi 1997).

Des Weiteren wird Stottern mit einer genetischen Disposition in Verbindung gebracht (Ambrose et al. 1997). Im Jahr 1939 konnte in einer Studie festgestellt werden, dass ca. die Hälfte der stotternden Personen stotternde Verwandte aufwiesen, während bei nicht stotternden Personen nur 18\% der Verwandten stottern (West et al. 1939). Stotternde Mütter oder Väter vererben ihre Sprechstörung eher an Söhne - mütterlicherseits: $36 \%$, väterlicherseits: $22 \%$ - als an Töchter - mütterlicherseits: 17\%, väterlicherseits: $9 \%$ - (Andrews et al. 1983). Aus diesen Daten kann man ebenfalls schließen, dass Mütter häufiger als Väter das Stottern an ihre Kinder vererben (von Müttern: 53\% an Söhne und Töchter, von Vätern: 31\% an Söhne und Töchter). Auch bei eineiigen Zwillingen kommt es häufiger vor, dass beide stottern als bei zweieiigen Zwillingen (Seemann 1937; Nelson et al. 1945; Andrews et al. 1991). 


\subsubsection{Remission}

Stottern tritt vorübergehend meist bis zur Pubertät auf (Natke 2005). Ob es zu einer spontanen Remission kommt, ist jedoch kaum vorherzusagen. Ein CIS besteht bei ca. $1 \%$ der Betroffenen fort, wobei mehr Jungen als Mädchen von einer Persistenz betroffen sind (Bloodstein 1995). Bei Mädchen tritt Stottern in einem jüngeren Alter auf, und es kommt früher zu einer Remission (Yairi 1983; Yairi und Ambrose 1992). So ergibt sich ein Verhältnis zwischen Jungen und Mädchen zu Beginn des Stottern von 2,6 zu 1 (Andrews und Harris 1964). Mit dem Heranwachsen verschiebt sich die Verteilung auf 3,6 zu 1 (Natke 2005). Bloodstein (1995) beschrieb sogar ein Verhältnis bei Jungen und Mädchen von 4 bis $5 \mathrm{zu}$ 1. In der Literatur finden sich unterschiedliche Angaben zum Anteil der Kinder, die eine Remission erfahren. Die Spannbreite liegt dabei zwischen 36,3\% und $78,9 \%$ (Natke 2005). Nach Andrews und Harris (1964) liegt die Remissionsrate sogar bei $79,1 \%$. Lattermann (2011) hatte die Forschungsergebnisse in den letzten Jahren zusammengefasst und Risikofaktoren für das Persistieren des Stotterns dargestellt. Die Remission des Stotterns hängt demnach von verschiedenen Faktoren wie Geschlecht, Beginn der Störung, familiärer Belastung, Alter, Verlauf der Störung, Leidensdruck des Kindes sowie phonologischer und sprachlicher Entwicklung ab.

\subsubsection{Auswirkungen des Stotterns auf die Lebensqualität}

Nach einer Studie von McAllister et al. (2012) beeinträchtigt das CIS die Lebensqualität und den sozioökonomischen Erfolg. Viele betroffene Personen empfinden ihre Sprechstörung als negativen Einfluss bei ihrer Beschäftigung. 71\% der stotternden Personen glauben, dass ihre Sprachstörung die individuellen Chancen für eine Einstellung verringert und $70 \%$ sind der Meinung, dass ihr Stottern ein Hindernis für die berufliche Förderung darstellt.

Stotternde Menschen mit einem hohen Schweregrad der Sprechstörung zeigen ein erhöhtes Risiko, an emotionaler Dysfunktion und dem sogenannten FatigueSyndrom zu erkranken, welches sich in Form von Stressgefühl, depressiver 
Verstimmung, Müdigkeit und Schläfrigkeit äußert (Tran et al. 2011). In einer Arbeit von St Clare (2009) wurden Messmethoden zur Erhebung von Unhelpful Thoughts and Beliefs about Stuttering (UTBAS) entwickelt, die sich aus den jahrelangen Verhaltenstherapien bei Stotternden kristallisierten. Das Ergebnis der Arbeit zeigte einen signifikanten Unterschied zwischen Stotternden und Flüssigsprechenden, wobei nicht hilfreiche Gedanken und Vorstellungen zu einer sozialen Angst bei Stotternden führen können. Da sich negative Einflüsse bereits im Kindesalter manifestieren ist es notwendig die Sprechstörung rechtzeitig zu erkennen und zu behandeln.

\subsection{Theorien zur Ätiologie des Stotterns}

\subsubsection{Geschichte}

Mindestens seit die Dokumentation der menschlichen Sprache existiert, ist Stottern als eine Störung des Redeflusses bekannt (Leon-Sarmiento et al. 2013). Bevor es neuronale Untersuchungen zur Erklärung des Stotterns gab, wurden verschiedene Pathologien wie eine Abnormität des Sprechorgans (Trockenheit der Zunge und abnormer Sprechapparat) als Ursachen des Stotterns vermutet. Auch psychologische Ursachen wurden angenommen und therapiert bis neu gewonnene Erkenntnisse diese Theorie nicht mehr ausreichend erklären konnten (Andrews et al. 1983).

\subsubsection{Neuropathophysiologische Ansätze}

Die Pathophysiologie des Stotterns wird nach Prins (1991) in drei Ebenen unterteilt. Die erste Ebene wird als die Basisstörung (impairment) der neurophysiologischen Fehlfunktion bezeichnet. Bei der zweiten Ebene handelt es sich um die hör- und sichtbare Symptomatik (disability). Die dritte Ebene beinhaltet die Behinderung (handicap), mit der die stotternden Personen zu leben haben. In dieser Arbeit wird der Hauptfokus auf die Basisstörung der ersten Ebene gerichtet. 


\subsubsection{Allgemeines}

Bis heute sind die Theorie zur Physiologie des flüssigen Sprechens und die Theorie zur Pathophysiologie des idiopathischen Stotterns umstritten (Salmelin et al. 2000; Alm 2004). Mit Hilfe von MRT-Untersuchungen konnten bei Personen mit $\mathrm{CIS}$ abnorme Gyrifizierungen des Broca-Areals sowie ein vergrößertes rechtes und linkes Planum temporale gefunden werden (Foundas et al. 2001). Diese Ergebnisse wurden durch funktionell-bildgebende Studien bestätigt (Brown et al. 2005). Es konnte gezeigt werden, dass bei stotternden Personen eine Überaktivität des motorischen Areals, einschließlich des M1, des SMA, des Gyrus cinguli und des Vermis zerebelli besteht. Des Weiteren wurde eine Abnormität des Operculum frontalis und der anterioren Inselrinde der rechten Hemisphäre beschrieben. Ferner konnte die auditorische Aktivität in der Hörrinde, welche während der Sprachverarbeitung notwendig ist, bei stotternden Personen nicht nachgewiesen werden (Fox et al. 1996; Brown et al. 2005). In diesem Zusammenhang ist bekannt, dass durch externe auditive Stimulation, z. B. Musik hören oder Silben-getaktetes Sprechen das Stottern reduziert werden kann (Salmelin et al. 2000; Büchel und Sommer 2004). Entsprechend wurde in zahlreichen Sprachmodellen die hohe Relevanz der auditiv-sensorischen Funktion bei der Sprachproduktion hervorgehoben (Hickok und Poeppel 2004; Friederici 2006; Guenther 2006; Rauschecker und Scott 2009; Friederici 2011; Hickok 2012). Aktuelle Studien beschäftigen sich mit neuronalen Verbindungen zwischen den sprachrelevanten Hirnarealen. In einer Studie mittels der MEG konnten Salmelin und Kollegen (2000) zeigen, dass flüssige Sprecher beim verzögerten Lesen von Wörtern zunächst eine erhöhte Aktivität von neuronalen Populationen im linken GFI zeigen, gefolgt von einer erhöhten Aktivität von neuronalen Populationen im M1. Da der linke GFI zur Sprechplanung herangezogen wird und der linke M1 die Ausführung der Sprechbewegungen steuert, handelt es sich um einen plausiblen Zeitverlauf des Aktivierungsmusters. Bei Personen, die stottern, ist dieser Ablauf bemerkenswerterweise umgekehrt. Hier zeigen zunächst die M1Areale eine gesteigerte Aktivität, gefolgt von einer Aktivitätszunahme im linken GFI. Diese Beobachtungen lassen darauf schließen, dass ein gestörter zeitlicher Ablauf in der Sprechplanung und die daraus resultierende motorische Sprechstörung die Ursache für das Stottern darstellen können. 
Bildmorphologische und neuropathologische Befunde wiesen eine fokale Verminderung der weißen Substanz, der angrenzenden Integrität zur sprachbezogenen linken Hemisphäre sowie ein Defizit der funktionellen Verbindungen zwischen dem linken inferioren Frontallappen und dem M1 nach (Sommer 2002; Chang et al. 2008; Watkins et al. 2008; Cykowski et al. 2010; Chang et al. 2011). Es ist nicht geklärt, wie diese strukturellen Erkenntnisse in die intermittierende Sprechunflüssigkeit übersetzt werden können. In einer früheren elektroenzephalographischen Studie wurde eine abnorme hemisphärische Dominanz bei stotternden Personen beschrieben (Moore und Haynes 1980).

\subsubsection{Theorie der Kompensationsmechanismen}

Bei der Produktion des unflüssigen Sprechens stotternder Personen wurde auf der linken Hemisphäre im Putamen, im ventralen Thalamus und im anterioren cingulären Kortex eine vermehrte Aktivität mittels der MEG aufgezeichnet, während sich beim flüssigen Sprechen vermehrte Aktivität in der rechten Hemisphäre zeigte (Braun et al. 1997; Salmelin et al. 2000). Diese Verlagerung in die rechte Hemisphäre kann eine Kompensation des strukturellen Defizits der linken Hemisphäre sein und eine pathophysiologische Funktionsstörung darstellen (Neumann et al. 2005).

\subsubsection{Abnormes Bereitschaftspotential}

Deecke et al. (1986) konnten das Bereitschaftspotential ca. zwei Sekunden vor dem Sprechakt auf beiden Hemisphären bei flüssigsprechenden Personen aufzeichnen. Während der letzten 100 bis 200 ms der Vorbereitungsphase wurde das Potential auf der linken Hemisphäre signifikant größer. Dies spricht dafür, dass die Aktivität während der Sprechvorbereitung auf beiden Hemisphären zu verzeichnen ist. 
In der Magnetenzephalographiestudie von Walla et al. (2004) konnte festgestellt werden, dass bei stotternden Personen die neuronale Aktivität während der fokussierten Vorbereitung auf den Sprechvorgang fehlt. Darüberhinaus konnte festgestellt werden, dass die Aktivität der Lippenbewegung bei den stotternden Personen 20 ms später erscheint (bei $270 \mathrm{~ms}$ ) als bei Flüssigsprechenden (bei $250 \mathrm{~ms})$.

\subsubsection{Stottern als Störung des Übergangs beim Sprechen}

Wenn man analysiert wann Stottern auftritt, so zeigt sich, dass die unwillkürlichen Sprechbewegungen wie Lautwiederholungen, Lautdehnungen oder das ungewollte Stoppen im Sprechfluss am Wortanfang bzw. am Übergang zwischen Sprachlauten auftritt. Wingate (2002) beschreibt einen unwillkürlichen Kontrollverlust beim Übergang von einem Wortbeginn in eine sich anschließende artikulatorische Konfiguration. Die Zeitpunkte während der Übergangsphase wurden u.a. in dieser Arbeit aufgezeichnet und untersucht. 


\section{Material und Methoden}

\subsection{Transkranielle Magnetstimulation}

\subsubsection{Theoretischer Hintergrund}

Die TMS ist eine nicht-invasive, schmerzlose Methode zur Stimulation des motorischen Kortex. Die Probanden berichteten bei der TMS-Applikation mitunter von vorübergehenden Kribbelparästhesien der Kopfhaut sowie von der akustischen Wahrnehmung eines "Klick"-Geräusches, welches durch die Verformung der Spulenleiter bei großen magnetischen Kräften entsteht. Die Stimulation des motorischen Kortex kann eine Muskelzuckung im kortikal repräsentierten Erfolgsorgan auslösen oder eine Willkürbewegung unterdrücken (Rothwell 1997). Die Entdeckung der TMS geht auf das Jahr 1985 in London zurück (Barker et al. 1985). Eine kontaktlose Reizung der Nerven und Neurone umschriebener Hirnareale konnte durch die TMS induziert werden. Ueno et al. (1988) entwickelten die erste fokale Magnetspule, die einen besonderen Beitrag für die weitere Forschung erbrachte. Im darauf folgenden Jahr wurde eine achtförmige Doppelspule (figure-of-eight coil oder twin coil) entwickelt und am motorischen Kortex des Menschen angewendet (Rösler et al. 1989). Mit dieser Entwicklung wurde eine möglichst fokale Reizwirkung erzielt (Siebner und Ziemann 2007).

TMS wird in der Forschung, Diagnostik und Therapie eingesetzt. Beim kortikalen Mapping werden Funktionen bestimmten motorischen Arealen zugeordnet. In der klinischen Routine hat die TMS ihren Stellenwert in der Diagnostik von demyelinisierenden Prozessen und Motorneuronerkrankungen, kann aber aufgrund der unzureichenden Spezifität allerdings nicht als ausschließliches Diagnoseverfahren angewandt werden. Therapeutische Verwendung fand die TMS bisher nur im Rahmen experimenteller Studien, u.a. bei depressiven Erkrankungen mittels repetitiver TMS (George et al. 1995). Studien an Schlaganfallpatienten zeigten eine partielle Besserung der motorischen und sprachlichen Fähigkeiten sowie der Aufmerksamkeit (Floel et al. 2004; Hummel und Cohen 2006). Die bisherigen Studienergebnisse waren heterogen und aufgrund der überwiegend kleinen Fallzahlen nicht repräsentativ. Entsprechend ist die TMS nicht als standardisierte Therapie etabliert (Lefaucheur et al. 2014). 


\subsubsection{Technische Grundlage}

Bei der TMS wird ein kurzes magnetisches Feld erzeugt, das biologisches Gewebe durchdringt und dort lokal einen elektrischen Stromfluss induziert, der zur Depolarisierung der Neurone führt. Dabei misst das Zentrum des induzierten Magnetfeldes ca. 2,1 Tesla. Das zu stimulierende Kortexareal hat eine Ausdehnung von ca. $1 \mathrm{~cm}^{3}$. Das entstandene motorisch evozierte Potential (MEP) wird mittels der EMG quantifiziert (Rothwell 1997).

\subsubsection{Nebenwirkungen und Sicherheitsaspekte}

Die Anwendung von TMS bei Menschen ist nicht risikofrei. Es können während einer TMS-Sitzung Nebenwirkungen sowohl elektrischer als auch magnetischer Ursachen auftreten. Bei technischen Komplikationen, meist durch Defekt der Isolation, haben die Hersteller der Geräte dafür Sorge zu tragen, dass die Geräte störungsfrei beim Anwender abgegeben werden oder im Störungsfall sich automatisch abschalten (Siebner und Ziemann 2007).

Es werden akute und anhaltende Nebenwirkungen der TMS beschrieben. Die akuten Nebenwirkungen, zu denen Kontraktionen der Gesichts-, Schläfen- oder Nackenmuskulatur oder akute Spannungskopfschmerzen gehören, sistieren unmittelbar nach der Applikation der Stimuli oder klingen nach einigen Stunden ab. Auch wurden akute kognitive Effekte beobachtet, welche aber binnen kurzer Zeit verschwinden. Unerwünschte Langzeiteffekte durch TMS-induzierte elektrische Stimulationen werden als unbedenklich betrachtet. Bislang wurde bei Personen mit einer normalen kortikalen Erregbarkeit das Risiko durch einzelne oder gepaarte Stimuli einen epileptischen Anfall auszulösen als gering eingestuft (George und Aston-Jones 2010). Dagegen können bei Personen mit einer abnormal erhöhten kortikalen Erregbarkeit wenige Stimuli einen epileptischen Anfall auslösen. Bei der repetitiven TMS kann in bestimmten Stimulationsmodi das Risiko der Anfallsauslösung erhöht werden (siehe Übersicht Siebner und Ziemann 2007, Kap. 4). 
Aufgrund der genannten möglichen Nebenwirkungen sollen Anwender der TMS die internationalen Sicherheitsbestimmungen nach Wassermann (1998) und Rossi et al. (2009) beachten, um den Probanden oder Patienten nicht zu schaden. Sollten alle Ausschlusskriterien (siehe Punkt 3.4) beachtet werden, so wird die TMS als risiko- und nebenwirkungsarm beschrieben. Die Untersuchungen mit TMS wurden in dieser Studie nach den von Wassermann (1996) bzw. von Rossi und Kollegen (2009) aufgestellten Sicherheitskriterien durchgeführt. Bislang konnten keine gesundheitlichen Komplikationen in den Laboren der Klinik für Klinische Neurophysiologie der Universitätsmedizin Göttingen durch die Anwendung von TMS festgestellt werden. Zur Gewährleistung der Sicherheit für die Probanden sollen nur Probanden an der Studie teilnehmen, die keine Implantate im Kopfbereich, Schrittmacher oder Objekte mit Risiken bei Kontakt mit Elektroden im Körper tragen. Des Weiteren soll bei bestimmten internistischen Erkrankungen, wie schwerer Hypertonie oder dekompensierter Herzinsuffizienz sowie psychischen Erkrankungen die TMS nicht angewendet werden (siehe Punkt 3.4). Die Probanden sollen vor und während TMS-Sitzungen keine zentralwirksamen Medikamente einnehmen.

\subsubsection{Spulenposition zur Bestimmung der Zungenrepräsentation}

In dieser Studie wurde zur Aktivierung der Zungenmuskulatur die monophasische Magnetstimulation durch eine acht-förmige Spule (figure-of-eight coil) mit einem Außendurchmesser von $70 \mathrm{~mm}$ und mit einem posterior-anterioren Stromfluss in der üblichen 45-Grad-lateralen-Spulenposition über dem motorischen Kortex verwendet (Kaneko et al. 1996; Di Lazzaro et al. 2004). Die Magnetspule wurde mit einer Hand tangential mit einem Winkel von 45 Grad vom Spulengriff zur Sagittalebene gehalten. Dabei wurde die Magnetspule in 0,5 cm Schritten auf der vermuteten Region der Zungenrepräsentation von lateral nach medial und von anterior nach posterior bewegt. Der M1 erstreckt sich auf einer Linie, die ungefähr vom Scheitelpunkt zum präaurikulärem Punkt am Ohr verläuft, also 1-13 cm lateral vom Vertex (Rödel et al. 2003). Wenn man die Repräsentationsstelle der Hand zur

Orientierung nimmt, befindet sich der hot spot der Zungenrepräsentation ca. 2 bis 
$3 \mathrm{~cm}$ anterior und 1 bis $2 \mathrm{~cm}$ lateral von der Handrepräsentation (Svensson et al. 2006).

Die MEPs von der Zunge wurden unter der Verwendung eines speziell angefertigten Mundstücks aus Silikon aufgezeichnet. An diesem löffelförmigen Mundstück wurden zuvor zwei Paare austauschbarer, klebbarer Elektroden befestigt. Die Kontaktfläche der Elektroden auf der Zunge betrug $5 \mathrm{~mm}$ x $10 \mathrm{~mm}$ in Längsrichtung und $20 \mathrm{~mm}$ sowie $25 \mathrm{~mm}$ in seitlichen Abständen und wurden voneinander entfernt fixiert (5 mm x $100 \mathrm{~mm}$, Viasys Neurocare, Höchberg, Deutschland) (siehe Abbildung 3). Anschließend wurde das Mundstück auf der Zunge so platziert, dass die Elektroden mit der Zunge in Kontakt treten, während die Untersuchung stattfand (Rödel et al. 2003; Neef et al. 2011). Die Probanden wurden gebeten das Mundstück bei geschlossenem Mund mit leichtem Druck auf der Zunge zu halten. Dabei sollte das Mundstück von außen festhalten und zwar mit der Hand, die sich ipsilateral zum transkraniellen Magnetstimulus befand (Neef et al. 2011).

\subsection{Studiendesign und Zielparameter}

In dieser Studie sollte mittels der TMS untersucht werden, ob Erwachsenen, die stottern im Vergleich zu flüssigsprechenden Probanden beim Sprechen signifikante Unterschiede in der Modulation der Erregbarkeit des linken und des rechten M1 der Zunge zeigen. Dazu wurden während der Sprechaufgaben einzelne TMS-Pulse über die kortikale Zungenrepräsentation jeweils einer Hemisphäre appliziert. Die MEPs wurden mittels der EMG von der ipsilateralen und der kontralateralen Zungenseite abgeleitet.

\subsection{1 hot spot der Zungenrepräsentation}

Zur Lokalisation der Zungenrepräsentation wurde ein löffelähnliches Mundstück aus Silikon mit Elektroden beklebt und auf der Zunge platziert (siehe Punkt 3.1.4). TMS-Pulse wurden über dem linken und rechten M1 der Zungenrepräsentation verabreicht und MEPs generiert. Zunächst wurde die Kopfhautoberfläche 
systematisch untersucht und jene Position, welche bei niedrigster Reizstärke noch beständig induzierbare maximale MEPs in der kontralateralen Zungenseite erbrachte als hot spot identifiziert. Das EMG-Signal sollte auf der kontralateralen Seite stabile Werte auf einer Skala zwischen 1,0 und -1,0 mV anzeigen.

Dabei sollen die Probanden die Zunge leicht gegen das Mundstück drücken (Muellbacher et al. 2001; Neef et al. 2011). Das Stimulusintervall betrug 6 Sekunden $( \pm 10 \%, \sim 0,2 \mathrm{~Hz})$. Nach ca. 50 applizierten TMS-Pulsen wurden die aufklebbaren Elektroden auf dem Mundstück gewechselt. Der regelmäßige Wechsel der Elektroden war notwendig, um eine bessere Leitbarkeit der Elektroden zu gewährleisten. Der Zeitaufwand für den Wechsel der Elektroden dauerte ca. 5 Minuten. Der optimale Reizort hot spot wurde mit einem wasserfesten Stift markiert, um die definierte Stelle für die folgenden Untersuchungen festzulegen. Dieses Verfahren wurde von Rödel et al. (2003) übernommen. In dieser Studie waren ca. 100 TMS-Pulse erforderlich, um den hot spot und die motorische Schwelle zu finden. Eine Modulation der motorischen Kortexerregbarkeit durch TMS-Pulse während des Experiments konnte bei dieser geringen Anzahl und der langsamen Frequenz der TMS-Pulse ausgeschlossen werden (Fitzgerald et al. 2006). Die MEPs der Zungenrepräsentation für den Untersuchungsablauf wurden jeweils im rechten und linken Motorkortex bestimmt. Dabei wurden die bilateralen EMGs der Zungenmuskulatur des jeweiligen Motorkortex abgeleitet.

\subsubsection{Motorische Schwelle}

Nach der Bestimmung des hot spot wurde zunächst die individuelle motorische Schwelle bei jedem Probanden bestimmt. Die motorische Schwelle wird definiert als die kleinstmöglichste Reizintensität, die erforderlich ist, um MEPs zu erzeugen. Es wurden überschwellige TMS-Pulse appliziert und anschließend die Stimulationsintensität gesenkt bis die minimale Intensität erreicht wurde, welche mindestens $100 \mathrm{mV}$ EMG-Amplituden erzeugte. Eine leichte Vorinnervation des Zungenmuskels war notwendig, um einen sicheren Kontakt der Zungenoberfläche und den Ableitelektroden sicherzustellen (siehe Übersicht Siebner und Ziemann 2007, Kap. 9). Die peak-to-peak-Amplitude der MEPs bei dieser Studie mussten 
deshalb größer als $100 \mathrm{mV}$ statt der üblichen $50 \mathrm{mV}$ und mindestens durch drei von sechs aufeinanderfolgenden Reizen erzeugt worden sein. Die ermittelte Reizstärke wurde als die motorische Schwelle definiert, welche bei jedem Probanden unterschiedlich ist.

\subsubsection{Elektromyographie}

Mit Hilfe der EMG kann die Aktivität motorischer Einheiten registriert werden. Diese wird entweder mit in den Muskeln eingestochenen Nadelelektroden oder mit Oberflächenelektroden erfasst. In der EMG werden die Summenaktionspotentiale aufgezeichnet und zur Analyse komplexer Bewegungen und ihrer Störungen herangezogen (Klinke et al. 2005). Im Rahmen dieser Studie saßen die Probanden in einem bequemen Liegestuhl, während die Aktivierung der Zungenmuskulatur durch visuelle Rückmeldung der EMG-Aktivität kontrolliert wurde. Die MEPs der Zungenmuskulatur wurden als EMG-Signale abgeleitet und quantifiziert (siehe Abbildung 3).

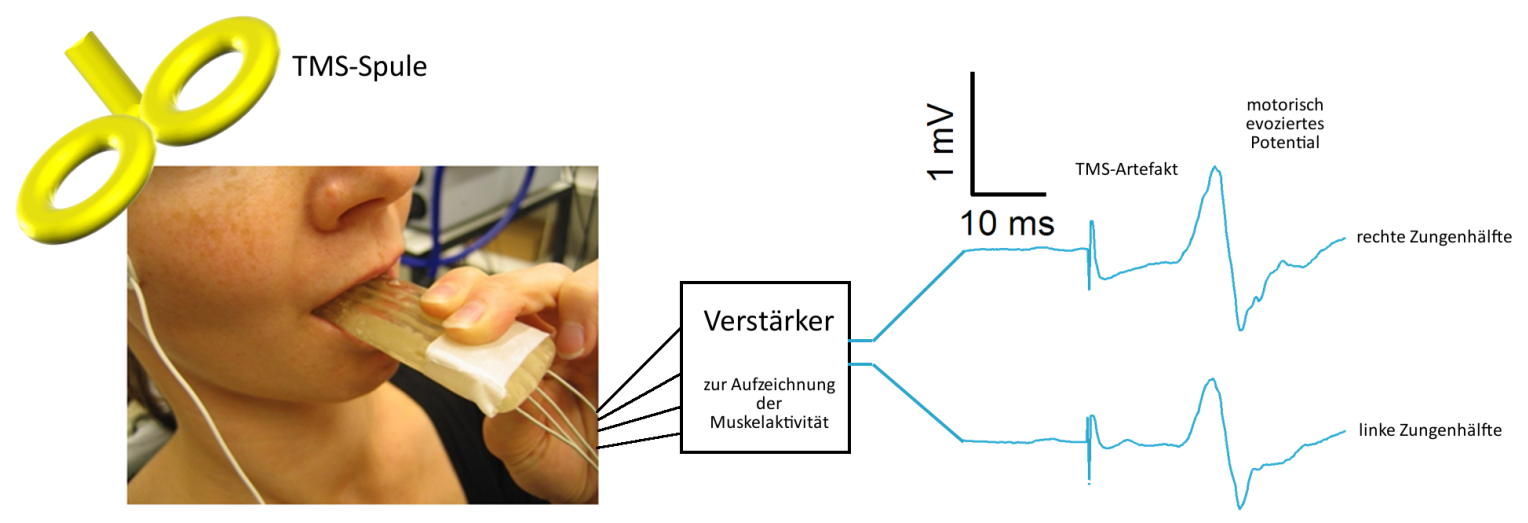

Abbildung 3: Versuchsaufbau mit EMG-Ableitungen. 


\subsubsection{Untersuchungsbedingungen}

Die Probanden saßen in einem bequemen Liegestuhl mit Kopf- und Armlehnen. Die einzelnen TMS-Pulse wurden mittels einer monophasischen Stromwellenform des Magstim $200^{2}$ magnetic Stimulators ${ }^{\circledR}$ appliziert (Magstim Company Ltd, Whitland, Wales).

Neben den Elektroden auf dem Mundstück zur Ableitung der EMG-Signale der Zungenrepräsentation wurde zur Erdung eine Elektrode von ca. $2 \times 3 \mathrm{~cm}$ Fläche (Veasys Neurocare, Höchberg, Deutschland) an die Stirn angebracht.

Die Signale wurden in einem Digitimer D $360^{\circledR}$-Verstärker (1000-fach) eingespeist (Digitimer Ltd, Welwyn Garden City, England) und von dort in einem CED 1401 $A / D^{\circledR}$-Konverter mit einer $5 \mathrm{kHz}$-Aufzeichnungsrate und einem Bandfilter, welcher Signale zwischen 20 und $2000 \mathrm{~Hz}$ filtert, aufgenommen. Die Daten wurden auf einem Computer unter Verwendung der Signal-Software (Cambridge Electronic Design, Version 2.13) erfasst und gespeichert. Es wurde zusätzlich ein leichtes Mikrofon (AKG PT 40, Wien, Österreich) an der Außenkante des Mundstücks befestigt, um den Spracheinsatz aufzunehmen. Die Ausgangssignale wurden direkt in einen dritten Kanal des A/D-Wandlers geleitet.

\subsubsection{Modulationsfähigkeit der motorisch evozierten Potentiale}

Um die funktionelle Integrität der motorischen Bahnen zu testen, wurde in einer Kontrollstudie die Verbindung zwischen dem M1 und der Zunge ohne Sprechaufgabe untersucht (Neef et al. 2015). Damit eine ausreichende dynamische Spannweite der Erregungsmodulation in beiden Gruppen gewährleistet und somit ein Ceiling-Effekt vermieden wird, wurden bei jedem Proband die MEP-Input-Output-Kurven mittels TMS-Pulsen auf dem optimalen Reizort (hot spot) mit ansteigender Intensität von 90, 100, 110, 120, 130, 140\% der individuell ermittelten motorischen Schwelle rekrutiert. Das EMG-Signal sollte Werte auf einer Skala zwischen 1,0 und $-1,0 \mathrm{mV}$ anzeigen. Jeweils 5 TMS-Pulse wurden pro Bedingung und insgesamt 30 auf jeder Hämisphäre separat 
verabreicht, um MEPs zu generieren, die in Form von EMG-Potentialen abgeleitet wurden.

\subsubsection{Ablauf der Untersuchung}

Die Probanden wurden in zwei verschiedenen Sitzungen zwischen denen mindestens 48 Stunden lagen auf beiden Hemisphären untersucht. Die Reihenfolge der getesteten Hemisphären wurden zwischen den beiden Probandengruppen randomisiert. In jeder Sitzung wurde mit der Messung der Baseline der Ruhemotorschwelle und der Input-Output-Kurve (Rekrutierung) begonnen. Anschließend erfolgte die Eingewöhnungszeit, in der die Probanden gebeten wurden, zehn Testversuche der Sprechaufgaben mit dem Mundstück durchzuführen. Während dieser Testversuche wurden keine TMS-Pulse verabreicht. In der eigentlichen Studie wurden insgesamt 98 Versuche durchgeführt, die in zwei Blöcke à 35 und einen Block à 28 Versuche aufgeteilt waren. Zwischen den Sprechblöcken wurde eine Pause für ca. fünf Minuten zum Wechseln der durch Speichel nicht mehr optimal abzuleitenden Elektroden eingelegt. Die Anordnung der 98 Verben wurde vom Computer randomisiert.

Die Liste von Wörtern mit dem Wortstamm für die Versuchsreihe wurde aus einer Datenbank ausgewählt. Diese sind in Hinblick auf Frequenz, phonetische Komplexität, Anzahl der Buchstaben, Höhe der Phoneme, Silben und Menge der Wortakzente geprüft worden (Webcelex.mpi.nl Webcelex, MPI Nijegen, NL).

\subsubsection{Bestimmung der Baseline}

Mit 120\% der individuell ermittelten motorischen Schwelle wurden 14 TMS-Pulse über dem M1 der Zungenrepräsentation, davon sieben TMS-Pulse vor dem Erscheinen des Verbs auf dem Computerbildschirm appliziert. Weitere sieben TMS-Pulse wurden nach dem Erscheinen des Verbs, während des Sprechens und Ausdehnens des Präfixes "auf" verabreicht. Das EMG-Signal sollte Werte auf einer Skala zwischen 1,0 und -1,0 mV anzeigen. Die Ermittlung der Baseline- 
MEPs dienen zur Evaluierung der Erregungsaktivität im Ruhezustand bevor die Sprechaufgabe beginnt, in der die Probanden aufgefordert werden, die Wörter zu lesen und in ihrem Gedächtnis zu speichern. Die generierten MEPs werden zum Vergleich mit den in den Sprechaufgaben ermittelten MEPs herangezogen.

\subsection{Experimenteller Aufbau}

\subsubsection{Sprechaufgaben}

Der neuronale Übergang von einer beginnenden auf eine anschließende artikulatorische Wortzusammenstellung wurde untersucht. Dabei wurde immer das gleiche Präfix "auf" als Wortanfang in der artikulatorischen Wortanordnung verwendet. Die Teilnehmer wurden in etwa $1 \frac{1}{2} \mathrm{~m}$ Entfernung vor einen Computerbildschirm gesetzt, und ihnen wurde eine Sequenz von Stimuli und Wörtern auf dem Bildschirm präsentiert.

Jede Sprechsequenz begann mit einem schwarzen Bildschirm. Nach $4000 \mathrm{~ms}$ erschien ein Wortstamm (z. B. "stehen"), welcher für für 1500 ms auf dem Bildschirm eingeblendet war, genug Zeit um das Wort zu lesen. Anschließend erschien für 3000 ms wieder ein schwarzer Bildschirm, ausreichend Zeit, um sich den Wortstamm zu merken. Hiernach erschien ein visuelles Go-Signal (in Form eines Kreuzes “+”) für 2000 ms, welches die Probanden aufforderte, das immer gleichbleibende Präfix "auf" zu sprechen und den labiodentalen Reibelaut zu verlängern („aufffff....) bis ein anderes visuelles Go-Signal angezeigt wird (in Form eines Ausrufezeichens “!”), welches für ca. 1500 ms auf dem Bildschirm erschien. Bei diesem Ausrufezeichen sollte der zuvor gezeigte Wortstamm (z. B. "stehen") - bei noch ausgedehntem labiodentalen Reibelaut des Präfixes "auf" ausgesprochen werden: „aufff...stehen“ (siehe Abbildung 4). 
$\mathbf{A}$
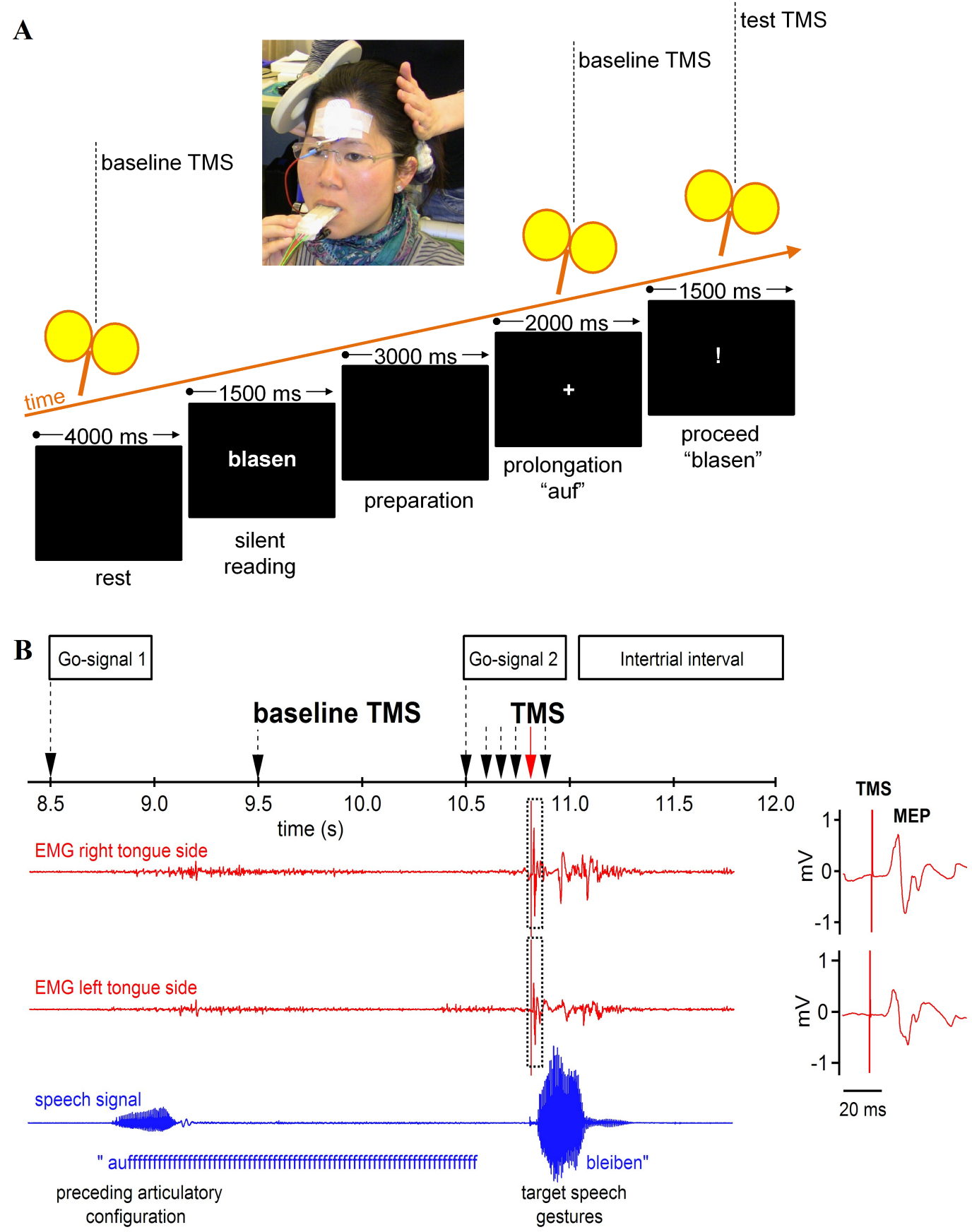

Abbildung 4: Versuchsablauf während einer Sprechaufgabe (publiziert in Neef, Hoang et al. 2015) 


\subsubsection{Aufzeichnungszeitpunkte}

Während der Sprechaufgaben wurden einzelne TMS-Pulse verabreicht. Diese wurden mit einem inter-trial-Intervall von 12 Sekunden appliziert. Die motorische Kortexerregung wurde zu sieben verschiedenen Zeitpunkten gemessen: (1) während der Ruhephase, 2900 ms nach dem Versuchsbeginn; (2) während der Ausdehnung der festen artikulatorischen Konfiguration des labiodentalen Reibelauts "f", 1000 ms nach dem ersten Go-Signal; (3) an fünf verschiedenen Zeitpunkten der Transitionsphase zwischen der festen Artikulationskonfiguration und der Zielbewegung, nämlich 240 ms, 280 ms, 320 ms, 360 und 400 ms nach dem zweiten Go-Signal. Insgesamt wurden 14 MEPs pro Bedingung für beide Hemisphären aufgezeichnet.

\subsection{Probanden}

Das Studienprotokoll wurde von der Ethikkommission der Georg-AugustUniversität Göttingen genehmigt. Alle Teilnehmer erhielten vor Beginn der Experimente ein ausführliches Aufklärungsgespräch über den Verlauf, die Risiken und den wissenschaftlichen Hintergrund der Studie. Nach ausreichender Bedenkzeit gaben die Probanden ihr schriftliches Einverständnis zur Teilnahme an der Studie. 17 Erwachsene, die stottern, wurden aus der StottererSelbsthilfegruppe in Göttingen und der Kasseler Stottertherapie rekrutiert. Stottern seit dem Kindesalter galt als Einschlusskriterium für Erwachsene, die stottern. Alters- und geschlechtsadaptiert wurden 16 flüssig sprechende Kontrollpersonen durch ein Inserat auf dem Campus der Georg-August-Universität Göttingen gewonnen. Flüssig sprechende Probanden durften keine bisherige Sprachstörung im Kindesalter aufweisen oder Verwandte haben, die stottern oder unter andere Sprechstörungen leiden. Die Kontrollgruppe wurde entsprechend Alter, Geschlecht und Bildung der Gruppe der stotternden Probanden angeglichen. Keiner der Probanden zeigte in der klinischen Routineuntersuchung neurologische oder internistische Pathologien. Alle Probanden wiesen ein normales oder korrigiertes Sehvermögen auf und keiner nahm zentralwirksame Medikamente 
während der Studie ein. Alle Probanden wurden den TMS-Test-Kriterien unterzogen (Keel et al. 2001).

Allgemeine Ausschlusskriterien für beide Gruppen:

- neurologische Erkrankung (insbesondere Epilepsie, Hirntumor, Migräne, Schlaganfall)

- Metallimplantate im Kopfbereich

- Herzschrittmacher

- im Körper enthaltene Objekte mit Risiken bei Kontakt mit Elektroden

- internistische Erkrankung (schwere Hypertonie, dekompensierte Herzinsuffizienz)

- psychiatrische Erkrankung (seelische Erkrankung, Erkrankung mit Wahn, Trugwahrnehmungen, Störungen des Denkens, Erkrankung des Gemüts mit gehobener Stimmung)

- Drogen-, Medikamenten- und /oder Alkoholabhängigkeit

- Störung des Sprachverständnisses und des Sprechens

- gleichzeitige Teilnahme an einer anderen Studie.

Zusätzliche Ausschlusskriterien für die Gruppe der stotternden Personen:

- Stottern durch traumatische Ereignisse mit einhergehenden Hirnschädigungen

Insgesamt wurden 33 Probanden für die Untersuchung gewonnen. Für drei Probanden war die individuell ermittelte motorische Schwelle zu hoch. Die zu applizierende Stimulusintensität (bis 140\% der ermittelten Schwelle) für die Rekrutierung wäre sehr hoch gewesen und hätte lokale Schmerzen ausgelöst. Drei weitere Probanden empfanden das Experiment in der ersten Studiensitzung als unangenehm und beendeten die Studienteilnahme. Bei der Sprachanalyse wies ein subjektiv flüssig sprechender Proband 5\% gestotterte Silben auf und wurde vom Datensatz ausgeschlossen. Demzufolge wurden 13 Erwachsene in die Untersuchung eingeschlossen, die seit ihrer Kindheit stotterten. In dieser Gruppe 
waren vier Frauen und neun Männer im Alter von 21-55 Jahren (Durchschnittsalter 34,5 Jahre; SD = 12,0 Jahre). Acht stotternde Erwachsene gaben eine familiäre Häufung des Stotterns an. Zwölf der dreizehn stotternden Erwachsenen waren Rechtshänder und einer Linkshänder (Oldfield 1971). Die Kontrollgruppe bestand aus 13 flüssig sprechenden Probanden mit keiner persönlichen und familiären Belastung bezüglich des Stotterns, darunter vier Frauen und neun Männer im Alter von 23-44 Jahren (Durchschnittsalter 30,1 Jahre; $S D=7,8$ Jahre), die alle Rechtshänder waren. Die Kontrollgruppe wurde nach Alter, Geschlecht und Bildung mit der Gruppe stotternden Erwachsenen angeglichen. Keiner der Probanden zeigte in der klinischen Routineuntersuchung neurologische oder internistische Pathologien. Alle 26 Probanden, die an der Studie bis zu Ende teilgenommen haben, haben die Untersuchung gut vertragen. Es trat gelegentlich leichtes Zucken der Augenbrauen bei einigen Probanden während der Identifizierung des hot spot auf.

\subsubsection{Sprechflüssigkeit der Versuchsteilnehmer}

Bei Erwachsenen, die stottern, wurde durch die Analyse mittels des StotterDiagnostikverfahrens (SSI-3-Overall Score) ein Mittelwert von 23,5 (SD = 8,9 Silben) verzeichnet. Dieser Mittelwert berücksichtigt die Anzahl gestotterter Silben, die Dauer eines Stotterereignisses und die Ausprägung von Mitbewegungen von Gesichtsmuskulatur und Extremitäten. Wird ausschließlich die Anzahl der gestotterten Silben berücksichtigt, so zeigen Erwachsene, die stottern, im Mittel $7,6 \%$ (SD = 6,0 Silben). Die Stichprobe der Erwachsenen, die stottern, ist durch folgende Verteilung des Schweregrades gekennzeichnet: sechs wiesen einen sehr geringen, einer einen geringen, zwei einen moderaten, drei einen schweren und einer einen sehr schweren Schweregrad des Stotterns auf. In der Kontrollgruppe wurden keine signifikanten Sprechunflüssigkeiten festgestellt (siehe Abbildung 2). 


\begin{tabular}{llll}
\hline Maße & Stotternde & Kontrollen & Unterschied \\
\hline Probanden (n) & 13 & 13 & $\mathrm{n} / \mathrm{a}$ \\
Frauen (n) & 4 & 4 & $\mathrm{n} / \mathrm{a}$ \\
Alter in Jahren & $34.5(7.8)$ & $30.2(12.0)$ & $p=0.3$ (n.s.) \\
Stotterbeginn (Jahren) & $4.0(2.1)$ & $\mathrm{n} / \mathrm{a}$ & $\mathrm{n} / \mathrm{a}$ \\
SSI-3 Gesamtwert & $23.5(8.9)$ & $\mathrm{n} / \mathrm{a}$ & $\mathrm{n} / \mathrm{a}$ \\
\% gestotterte Silben & $7.6(6.0)$ & $0.3(0.2)$ & $p=0.001$ \\
Händigkeit & $75.2(43.6)$ & $87.6(17.8)$ & $p=0.35$ (n.s.) \\
Bildung* & 3 & 4 & $p=0.09$ (n.s.) \\
Motor. Schwelle li Hemisphäre & $41.9(7.3)$ & $37.6(3.2)$ & $p=0.07$ (n.s.) \\
Motor. Schwelle re Hemisphäre & $42.6(5.6)$ & $37.7(3.4)$ & $p=0.01$ \\
\hline
\end{tabular}

Tabelle 2: Charakteristische Eigenschaften der Versuchsgruppen.

*Bildung wird berichtet als Median, Gruppenunterschiede wurden mittels MannWhitney-U-Tests geprüft. SSI-3 = Stuttering Severity Instrument, dritte Ausgabe; $\%$ gestotterte Silben = gestotterte Silben pro 100 Silben; n.s. = nicht signifikant. $\mathrm{li}=$ links, $\mathrm{re}=$ rechts (modifiziert nach Neef, Hoang et al. 2015)

\subsubsection{Messung der Sprechflüssigkeiten}

Alle Teilnehmer waren deutsche Muttersprachler. Die Beurteilung der Sprechflüssigkeit wurde mit der Deutschen Version des SSI-3, Stuttering Severity Instrument (Sandrieser und Schneider 2008) durchgeführt. Die Sprechproben wurden mit Video aufgenommen während die Probanden über ihre aktuellen Aktivitäten (z. B. Arbeit, Schule oder Studium) sowie von ihrem Alltag berichteten. Die Sprechproben enthielten 500 Silben für eine Unterhaltung und mindestens 340 Silben für die Vorleseaufgabe (Neef et al. 2011). Die Videoaufnahmen der Sprechproben wurden durch eine qualifizierte Logopädin (N.E. Neef) ausgewertet. Dabei wurden Verlängerungen, Blockierungen und Geräusche als stotternde Silben gezählt. Um den Schweregrad des Stotterns zu beurteilen, wurden wiederholte einsilbige Wörter mit inadäquatem Auftreten von Stress und Nervosität gezählt. Sowohl die geschätzte Dauer der drei längsten Sprachblöcke als auch die körperlichen Begleiterscheinungen wurden bei der Bewertung des Schweregrades 
des Stotterns mit eingeschlossen (Neef et al. 2011). Die Deutsche Version der SSI-3-Normen wurde für die Beurteilung des Stotterschweregrades angepasst (Riley GD 1994; Neef et al. 2011). Alle stotternden Erwachsenen zeigten in den Sprechproben Kernsymptome des Stotterns wie Wiederholungen und Verlängerungen der Geräusche.

\subsubsection{Zuordnung der Händigkeit}

Da bei $90 \%$ der Rechtshänder das Sprachzentrum auf der linken Hemisphäre liegt, ist es für die Studie wichtig, die Händigkeit der Probanden zu bestimmen. Alle Probanden erhielten vor dem Versuch einen Fragebogen zur Erfassung der Händigkeit nach dem Edinburgh-Händigkeitsinventar-Protokoll (Oldfield 1971). In diesem Protokoll sind zehn manuelle Aktivitäten (z. B. schreiben, zeichnen, werfen) aufgelistet mit zwei Spalten, die Rechts- bzw. Linkshändigkeit für die Probanden zum Ankreuzen anbieten. Je nachdem, welche Hand die Probanden für die vorgegebene Tätigkeit vorzogen, setzten sie ein “+” in die entsprechende Spalte ein. Ist die Präferenz für die eine Händigkeit so stark, dass ausschließlich nur die eine Hand verwendet wurde, werden zwei “++" in diejenige Spalte gesetzt. Für den Fall, dass die Probanden sich nicht für eine Händigkeit entscheiden können, sollte jeweils ein “+” in beiden Spalten platziert werden. Zur Bewertung der Händigkeit wurden für die Kreuze Punktwerte vergeben. Wurde die Tätigkeit ausschließlich mit links ausgeübt (linke Spalte: "++") erhielt das Ergebnis für diese Tätigkeit -2. Für Tätigkeiten, die sowohl mit links als auch rechts durchgeführt werden, beträgt das Ergebnis 0. Zwei Punkte (+2) erhielt das Ergebnis, wenn die Tätigkeit nur mit rechts bewerkstelligt wurde (rechte Spalte: "++"). Der Lateralisierungsquotient ergab sich aus der Summe der Punkte der rechten und linken Spalte. Anschließend wurde dieser durch die maximale Gesamtpunktzahl dividiert und mit 100 multipliziert. Der Lateralisierungsquotient kann dabei -100 (ausschließlich Linkshänder) bis +100 (ausschließlich Rechtshänder) betragen. Probanden, die einen Lateralisierungsquotienten von $>+50$ bzw. $<-50$ aufwiesen, gelten als Subgruppe der Rechts- bzw. Linkshänder. Bei einem Lateralisierungsquotienten zwischen +50 und -50 wurden Probanden als Beidhänder zugeordnet. 


\subsection{Datenanalyse}

\subsubsection{Auswertung der Messergebnisse}

Die erfassten und gespeicherten MEPs wurden unter Verwendung der SignalSoftware (Cambridge Electronic Design, Version 2.13) graphisch dargestellt. Aus den Rohdaten wurden mittels visuell gesetzter Marker die MEP-Amplituden (peakto-peak-Amplituden) durch den individuell geschriebenen EMG-Browser in Igor Pro $^{\circledR}$ (Wavemetrics, USA, Dr. Andreas Neef) bestimmt. Um Prästimulus-EMGs der Zungenaktivität herauszufiltern, die vor den TMS-Pulsen entstehen, wurde das elektromyographische Signal aller Aufnahmen einzeln visuell ausgewertet. Zusätzlich wurden Signale mit TMS-Artefakten ausgeschlossen, die außerhalb der Motor-evozierten Antworten lagen. Mittlere peak-to-peak-Amplituden der kontralateralen und ipsilateralen Projektionen wurden für jede Bedingung berechnet. Die akustischen Latenzen wurden offline extrahiert, um den Sprechbeginn mit der Zungenbewegung in Beziehung zu setzen. Dabei wurde der reaktionsgekoppelte Zeitverlauf der Erregbarkeitsmodulation bestimmt. Der Sprechbeginn wurde als Zeitpunkt Null festgesetzt. Die MEP-Amplituden wurden über sieben aufeinanderfolgende Zeitfenster von jeweils $50 \mathrm{~ms}$ gemittelt, von -360 ms vor Sprechbeginn bis $40 \mathrm{~ms}$ nach Sprechbeginn. Über eine Beobachtungsphase von $400 \mathrm{~ms}$ ergab das acht Messzeitpunkte. Für jedes Zeitfenster in der Gruppe der Stotternden und Flüssigsprechenden wurden mindestens neun Beobachtungswerte zur Berechnung herangezogen.

\subsubsection{Statistische Analyse}

Die demographischen, verhaltens- und neurobiologischen Parameter für Alter, Händigkeit, Bildung, Gesamtbewertung des Stotterschweregrades, Prozentsatz der gestotterten Silben und motorische Schwelle wurden in Kolmogorov-SmirnowTests für jede Gruppe gesondert berechnet, um zu bewerten, ob beide Gruppen aus einer Population mit einer bestimmten Verteilung kommen (Neef et al. 2015). Mit dem zweiseitig ungepaarten $t$-Test (two-tailed unpaired $t$-test) wurden die Mittelwertunterschiede zwischen den stotternden und flüssigsprechenden 
Personen aus Alter, Händigkeit, Prozentsatz der gestotterten Silben und motorischer Schwelle überprüft.

Die Signifikanz von Gruppenunterschieden in Schulbildung, Sprecheinsatzzeit und Zungenaktivität der pre-TMS während der Baseline und der Ausdehnung des Präfix „auf“ wurden mittels des Mann-Whitney-Tests untersucht (siehe Tabelle 2).

Eine mixed-model ANOVA mit Zwischensubjektfaktor "Gruppe" (between-subjectfactor) und „Hemisphären“ (links, rechts) sowie „Zungenprojektion“ (ipsilateral, kontralateral) als Innersubjektfaktor (within-subject factor) wurde herangezogen, um die Varianz der motorischen Kortexerregbarkeit beim Sprechen und Ausdehnen des deutschen Präfix „auf“ zu testen.

Die Gruppenunterschiede der sprachspezifischen Modulation der motorischen Kortexerregbarkeit wurden mit dem zweiseitig ungepaarten $t$-Test überprüft. Die ermittelten MEPs wurden mit den normierten MEP-Amplituden der BaselineErregbarkeit, die während des Aussprechens des labiodentalen Reibelauts entstanden sind, verglichen. Die Unterschiede zwischen der sprechaufgabenbezogenen Erregbarkeit und der Baseline-Erregbarkeit (baseline-excitability) wurden mittels zweiseitig ungepaartem $t$-Test überprüft.

Aufgrund der individuell variablen Verzögerung zwischen Go-Signal und SprechEinsatz erhielten nicht alle Zeitfenster (bins) Daten von allen Probanden (siehe Abbildung 5). Der Mittelwert der normierten MEP-Amplituden über alle Zeitklassen (time bins) bei stotternden Personen wurde mit dem Prozentsatz der gestotterten Silben mit einer Exponentialfunktion ins Verhältnis gesetzt. Dabei wurden die MEPs für beide Hemisphären und ihre zugehörigen Zungenprojektionen zum Vergleich herangezogen.

Eine mixed-model ANOVA wurde verwendet, um die Varianz der motorischen Schwelle (stotternde Erwachsene, flüssig sprechende Probanden) als Zwischensubjektfaktor "Gruppe“ (between-subject factor) und den Hemisphären „links, rechts“ als Innersubjektfaktor (within-subjec factor) zu testen. Die Varianz der MEP-Input-Output-Kurven wurde mit einer mixed-model ANOVA als Zwischensubjektfaktor (between-subject factor) „Gruppe“ und „Hemisphären“ (links, rechts), „Zungenprojektion“ (ipsilateral, kontralateral) und „Intensität“ (90, $100,110,120,130,140 \%$ der motorischen Schwelle als Innersubjektfaktoren (within-subject factors) getestet. 


\section{Ergebnisse}

\subsection{Allgemeines}

Die Messung der MEP-Amplituden dient als Maß der Modulation der Erregbarkeit des sprechmotorischen Kortex. Diese Erregbarkeit wurde zu verschiedenen Zeitpunkten gemessen - während der Baseline, d. h. während der Artikulation des labiodentalen Frikatives [f] in "auf“ (siehe Punkt 4.2), und während der anschließenden Übergangsphase zum Sprechbeginn des Zielwortes, z. B. „bleiben“ (siehe Punkt 4.3).

4.2 Neuronale Fazilitation der primär motorischen Zungenrepräsentation während der kontrollierten Prolongation des Reibelauts [ $\mathrm{f}$ ]

Zur Untersuchung der neuronalen Fazilitation während der kontrollierten Verlängerung des labiodentalen Reibelauts werden die MEP-Amplituden mit denen der Aktivität im Ruhezustand (baseline-activity) normiert. Abbildung 5 zeigt für flüssig sprechende Probanden eine zerebral rechts-lateralisierte Erhöhung der Erregung in der linken Zungenseite. Im Kontrast dazu zeigen stotternde Erwachsenen eine zerebral links-lateralisierte Erhöhung der Erregung in der kontralateralen Zungenseite (siehe Abbildung 5). Dieses Ergebnis zeigte sich in der Interaktion von Gruppe und Hemisphäre $(F(24.1)=5.0, p=0.036$, Greenhouse-Geisser-Korrektur). Post hoc $t$-Tests bestätigten einen Trend zu einer erhöhten Erregung der rechtskortikalen Zungenrepräsentation bei flüssig sprechenden Probanden ( $t=2.06, p=0.062)$ und eine signifikant erhöhte Erregung der linkenskortikalen Zungenrepräsentation bei stotternden Erwachsenen $(t=2.43, p=0.032)$. 

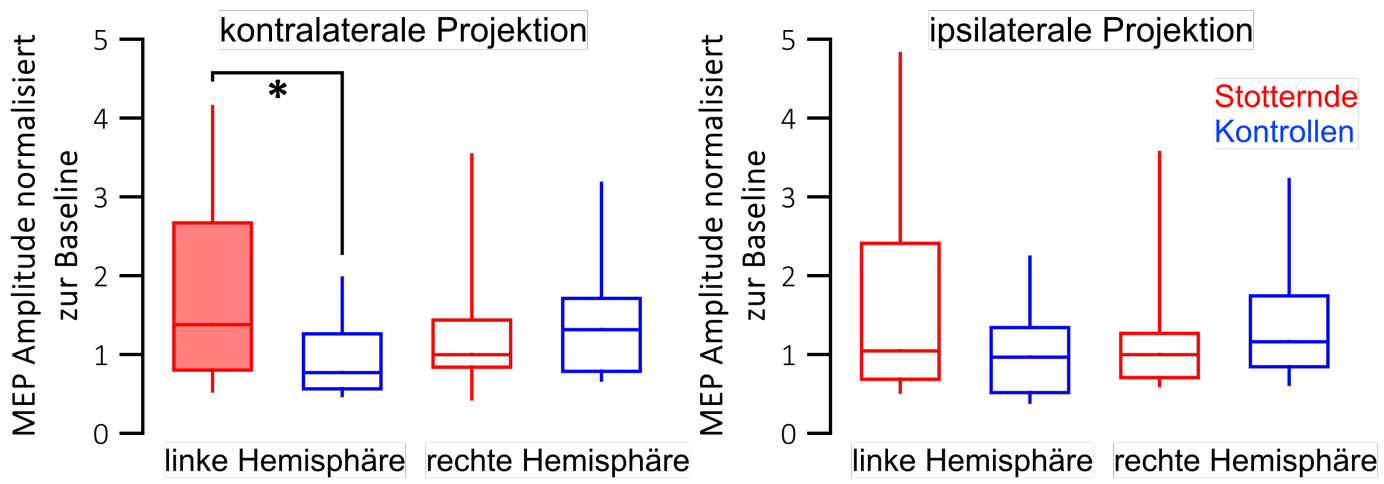

Abbildung 5: Die Dehnung des Reibelautes [f] führt zu einer erhöhten Erregbarkeit des linken Sprechmotorkortex bei Erwachsenen, die stottern. Dargestellt ist jeweils der gemittelte Gruppenwert der MEP-Amplitude normalisiert zur MEP-Amplitude während Ruhe (Baseline), (modifiziert nach Neef, Hoang et al. 2015)

4.3 Neuronale Fazilitation der primär motorischen Zungenpräsentation in der Übergangsphase zum Sprechen des Zielwortes

Ermittelt wurden die MEP-Amplituden während der Übergangsphase - sprich in der Phase zwischen der festen Artikulationskonfiguration und der anschließenden Sprechbewegung - um zu testen, ob die Erregbarkeit der sprechmotorischen Planung moduliert wurde. Anschließend werden diese MEP-Amplituden, an den Stimulus-gekoppelten Zeitpunkten, im Verhältnis zu MEP-Amplituden während der Verlängerung des Reibelauts "ffff" normiert.

Bei den flüssig sprechenden Probanden wurde bei der Stimulation der linken Hemisphäre sowohl für die kontra- als auch für die ipsilaterale Zungenseite eine signifikant erhöhte Erregbarkeit ab $260 \mathrm{~ms}$ vor Sprechbeginn des Zielwortes verzeichnet $(0.0095<p<0.049$, unkorrigiert; siehe Abbildung 6 und Tabelle 3$)$.

Bei der Stimulation der rechten Hemisphäre konnte dagegen keine neuronale Fazilitation in der kontralateralen Zungenprojektion während der Übergangsphase in die nachfolgenden Sprechbewegungen beobachtet werden. Ausnahme bildet 
die Erregbarkeitssteigerung der ipsilateralen Zungenprojektion zum Zeitpunkt 110 ms bis 60 ms vor Sprechbeginn ( $p=0.039$, unkorrigiert).

Stotternde Erwachsene zeigten keine Fazilitation der Neuronen der primär motorischen Zungenrepräsentation während der Stimulation der linken Hemisphäre (siehe Abbildung 6 und Tabelle 3).

Um Unterschiede zwischen stotternden Erwachsenen und flüssig sprechenden Probanden zu überprüfen, wurden zweiseitige ungepaarte $t$-Tests verwendet. Demzufolge zeigten flüssig sprechende Probanden eine signifikant erhöhte Erregbarkeitssteigerung für die kontralaterale Zungenprojektion während der Stimulation der linken Hemisphäre ab -160 ms vor Sprechbeginn $(0.004<p<$ 0.041 unkorrigiert, siehe Tabelle 4). 

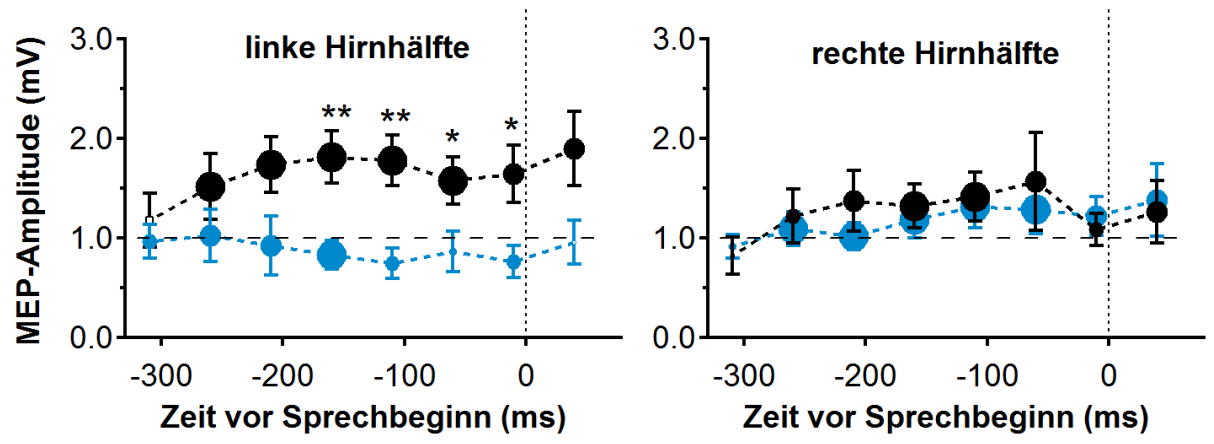

Kontrollen

Stotternde

Abbildung 6: Darstellung der Erregbarkeitsprofile der rechten und linken sprechmotorischen Zungenrepräsentation während des Sprechens (modifiziert nach Neef, Hoang et al. 2015)

\begin{tabular}{llllllllll}
\hline & \multicolumn{3}{l}{ Stotternde Erwachsene } & \multicolumn{3}{l}{ Kontrollen } \\
& \multicolumn{2}{l}{ linke Hemisphäre } & rechte Hemisphäre & \multicolumn{2}{l}{ linke Hemisphäre } & \multicolumn{2}{l}{ rechte Hemisphäre } \\
& Contra & Ipsi & Contra & Ipsi & contra & ipsi & contra & ipsi \\
\hline$-310-260 \mathrm{~ms}$ & 0.830 & 0.700 & 0.364 & 0.517 & 0.537 & 0.978 & 0.489 & 0.997 \\
$-260-210 \mathrm{~ms}$ & 0.921 & 0.216 & 0.439 & 0.635 & 0.145 & $\mathbf{0 . 0 1 8}$ & 0.604 & 0.812 \\
$-210-160 \mathrm{~ms}$ & 0.807 & 0.292 & 0.238 & 0.403 & $\mathbf{0 . 0 2 1}$ & $\mathbf{0 . 0 2 0}$ & 0.921 & 0.835 \\
$-160-110 \mathrm{~ms}$ & 0.257 & 0.503 & 0.167 & 0.466 & $\mathbf{0 . 0 0 9}$ & $\mathbf{0 . 0 1 3}$ & 0.333 & 0.166 \\
$-110-60 \mathrm{~ms}$ & 0.122 & 0.166 & 0.116 & 0.271 & $\mathbf{0 . 0 0 9}$ & $\mathbf{0 . 0 0 5}$ & 0.173 & $\mathbf{0 . 0 3 9}$ \\
$-60-10 \mathrm{~ms}$ & 0.508 & 0.237 & 0.272 & 0.749 & $\mathbf{0 . 0 3 4}$ & $\mathbf{0 . 0 1 5}$ & 0.261 & 0.610 \\
$-10-40 \mathrm{~ms}$ & 0.176 & 0.360 & 0.621 & 0.769 & $\mathbf{0 . 0 4 9}$ & $\mathbf{0 . 0 2 3}$ & 0.293 & 0.633 \\
$40-90 \mathrm{~ms}$ & 0.850 & 0.068 & 0.428 & 0.436 & $\mathbf{0 . 0 3 5}$ & $\mathbf{0 . 0 3 0}$ & 0.318 & 0.593 \\
\hline
\end{tabular}

Tabelle 3: $p$-Werte der zweiseitig gepaarten $t$-Tests zur Prüfung der sprechinduzierten Erregbarkeitssteigerung. Signifikante Werte sind fettgedruckt. ipsi $=$ ipsilaterale Projektionen; contra $=$ kontralaterale Projektionen (modifiziert nach Neef, Hoang et al. 2015) 


\begin{tabular}{lllll}
\hline & \multicolumn{2}{l}{ linke Hemisphäre } & \multicolumn{2}{l}{ rechte Hemisphäre } \\
& Contra & Ipsi & contra & ipsi \\
\hline$-310-260 \mathrm{~ms}$ & 0.508 & 0.275 & 0.656 & 0.674 \\
$-260-210 \mathrm{~ms}$ & 0.261 & 0.722 & 0.689 & 0.806 \\
$-210-160 \mathrm{~ms}$ & 0.056 & 0.383 & 0.269 & 0.383 \\
$-160-110 \mathrm{~ms}$ & $\mathbf{0 . 0 0 4}$ & 0.068 & 0.648 & 0.111 \\
$-110-60 \mathrm{~ms}$ & $\mathbf{0 . 0 0 2}$ & 0.187 & 0.752 & 0.878 \\
$-60-10 \mathrm{~ms}$ & $\mathbf{0 . 0 4 1}$ & 0.522 & 0.609 & 0.956 \\
$-10-40 \mathrm{~ms}$ & $\mathbf{0 . 0 1 7}$ & 0.452 & 0.611 & 0.587 \\
$40-90 \mathrm{~ms}$ & 0.061 & 0.387 & 0.803 & 0.649 \\
\hline
\end{tabular}

Tabelle 4: $p$-Werte der zweiseitig ungepaarten $t$-Tests zur Prüfung der Gruppenunterschiede zwischen Erwachsenen die stottern und Kontrollen hinsichtlich der Erregbarkeitssteigerung. Signifikante Werte sind fettgedruckt. ipsi = ipsilaterale Projektionen; contra = kontralaterale Projektionen (modifiziert nach Neef, Hoang et al. 2015)

4.4 Die Stotterschwere korrelierte mit der Erregbarkeitssteigerung des linken sprechmotorischen Kortex

Zusätzlich wurde für die stotternden Erwachsenen der Zusammenhang zwischen dem Schweregrad des Stotterns und der Modulation der Erregbarkeit getestet. Für die linkshemisphärische primärmotorische Repräsentation der Zunge zeigte sich ein negativer nichtlinearer Zusammenhang, der durch einen exponentiellen Verlauf gekennzeichnet ist (siehe Abbildung 7). Exponentielle Regressionen ergaben ein $R^{2}$ von $0.55(p=0.004)$ für die kontralaterale Projektion und ein $R^{2}$ von $0.30(p=0.055)$ für die ipsilaterale Projektion. 

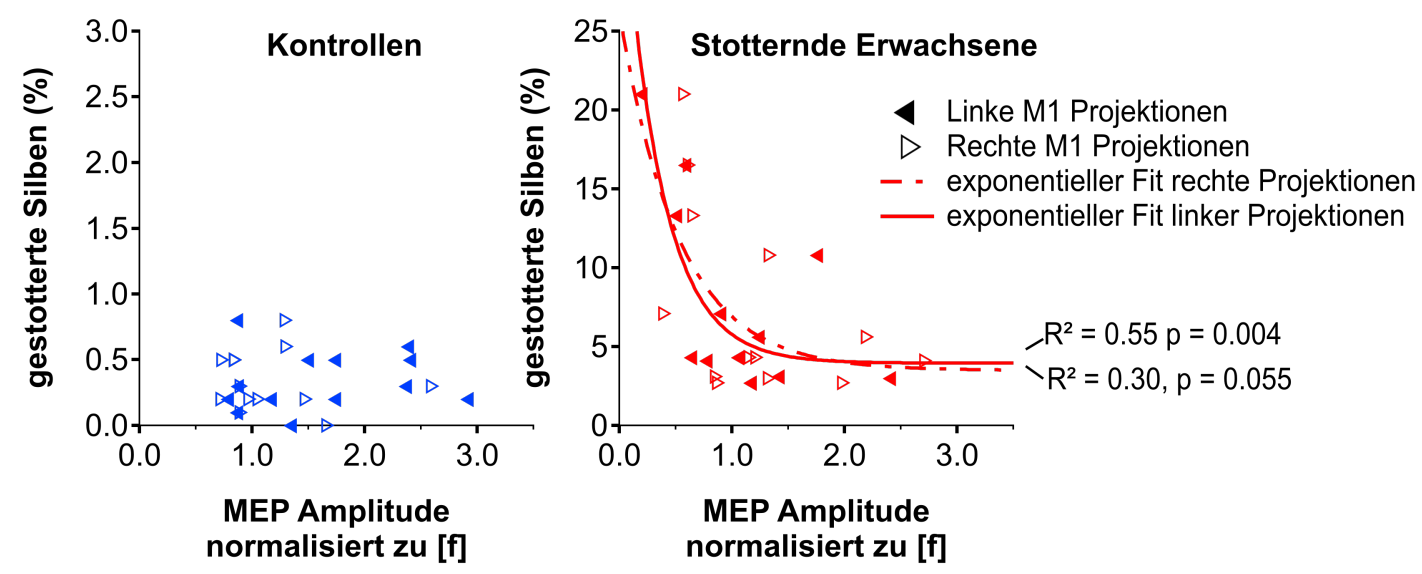

Abbildung 7: Negative Korrelation zwischen Schwergrad des Stotterns und Fazilitation des linken Sprechmotorkortex (modifiziert nach Neef, Hoang et al. 2015)

4.5 Erregbarkeit der primär motorischen Zungenrepräsentation in Ruhe gemessen als motorische Schwelle

Die Erhebung der individuellen motorischen Schwellen war notwendig, um die individuelle Stimulationsintensität für das Sprechexperiment zu bestimmen. Die motorische Schwelle wird in Prozent der Intensität des maximalen StimulatorOutputs (\%MSO) angegeben. Kontrollprobanden zeigten für die linke Hemisphäre eine motorische Schwelle von 37,6 \%MSO (SD = 3,2 \%MSO) und für die rechte Hemisphäre eine motorische Schwelle von 37,7 \%MSO (SD = 3,4 \%MSO). Stotternde Erwachsene zeigten für die linke Hemisphäre eine motorische Schwelle von $41,9 \% \mathrm{MSO}$ (SD = 7,3 \%MSO) und für die rechte Hemisphäre eine motorische Schwelle von 42,6 \%MSO (SD = 5,6 \%MSO). Eine zweifaktorielle Varianzanalyse ergab einen Gruppenunterschied hinsichtlich dieser Motorschwelle (ANOVA, $F(1,24)=6.09, p<0.021)$. Die ANOVA ergab keinen Effekt der Hemisphären und keine Interaktion zwischen Hemisphäre und Gruppe. Stotternde Erwachsenen wiesen eine höhere Motorschwelle auf als flüssig sprechende Probanden $(\mathrm{t}(50)=-3.33, \mathrm{p}=0.0016$ mit Bonferroni-Korrektur). 
4.6 Erregbarkeit der primär motorischen Zungenrepräsentation in Ruhe gemessen als Input-Output-Kurve

Die MEP-Input-Output-Kurven wurden untersucht um die dynamische Breite der Erregbarkeitsmodulation in beiden Gruppen zu messen. Das Ergebnis der InputOutput-Kurve (siehe Abbildung 8) ergab einen Haupteffekt der Zungenprojektion $($ ANOVA, $F(1,23)=10.15, p=0.004)$, einen Haupteffekt der Intensität $(F(5,19)=$ $33.1, p<0.0001)$ und eine Interaktion der Zungenprojektion mit der Intensität $(F(5,19)=4.5, p=0.028)$, aber keinen Effekt der Gruppe $(F(1,23)=0.01 ; p=$ 0.91 ) und keine anderen Effekte. Die MEP-peak-to-peak-Amplituden wurden in beiden Gruppen mit ansteigender Stimulationsintensität größer, insbesondere in der kontralateralen Zungenprojektion nach der Stimulation der linken Hemisphäre (siehe Abbildung 8).

Der Effekt der Intensität ist wichtig für die Beurteilung der beobachteten Erregbarkeitssteigerung im Sprechexperiment. Während der Sprechaufgaben wurde mit einer Intensität stimuliert, die 120\% der Ruhemotorschwelle (RMS)

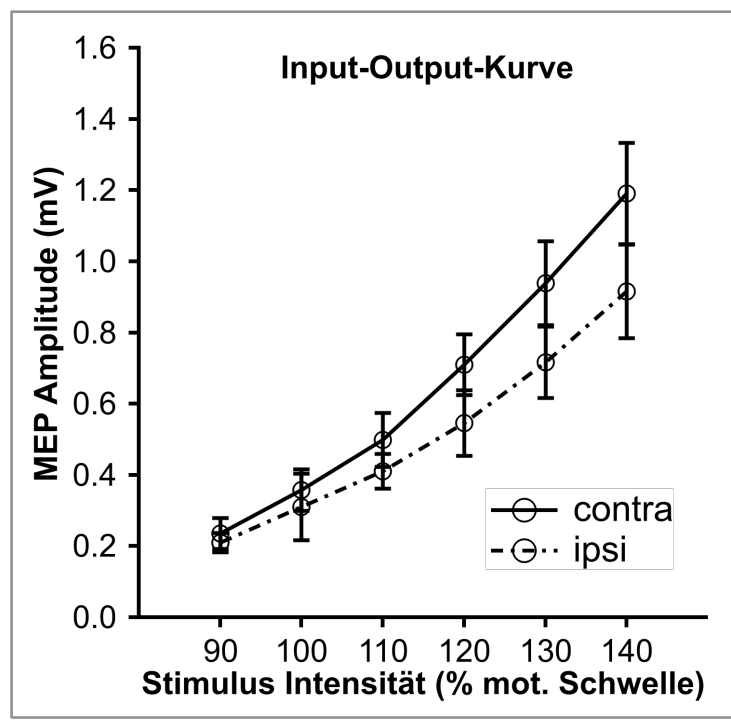

einer Versuchsperson beträgt. Da beide Gruppen bei einer Stimulationsintensität von $120 \%$ der RMS noch keine Sättigung zeigen, kann davon ausgegangen werden, dass die dynamische Breite der Erregbarkeitsmodulation noch nicht ausgeschöpft ist. Grundsätzlich ist demzufolge eine aufgabenbezogene Steigerung der Erregbarkeit in beiden Gruppen möglich.

Abbildung 8: Input-Output-Kurve für das Erregbarkeitsprofil des sprechmotorischen Kortex. Dargestellt sind die mittleren MEP-Amplituden für die kontralaterale und die ipsilaterale Projektionen des linken Sprechmotorkortex, gemittelt über beide Gruppen (modifiziert nach Neef, Hoang et al. 2015) 


\section{Diskussion}

Die Ursache des Stotterns ist immer noch nicht vollständig geklärt, obwohl es viele, u. a. bildgebende Studien gibt, die die Pathophysiologie des Stotterns zu erklären versuchen. In zahlreichen Studien ist die Dysfunktion des linken Motorkortex bei stotternden Personen beschrieben worden, jedoch fehlt es an grundlegenden Erklärungen dieser Dysfunktionen (Fox et al. 1996; Braun et al. 1997). Zugleich wurde die Überaktivität des rechten Motorkortex als Kompensationsmechanismus des dysfunktionellen linken motorischen Kortex bei stotternden Personen diskutiert. Insbesondere sind u. a. die afferenten Neuronen im linken Motorkortex bzw. die sprachrelevanten Bereiche des Gehirns untersucht worden. Die TMS dient als Untersuchungsmethode zur Beurteilung der Funktionsfähigkeit der kortikalen und kortiko-nukleären Bahnen des menschlichen Kortex (Tergau et al. 2001; Neef et al. 2011).

In den letzten Jahren gewinnt die TMS in der Diagnostik und Therapie neuropsychiatrischer Störungen immer mehr an Bedeutung (Paulus 2009). Mittels der TMS werden in den Laboren der Klinik für Klinische Neurophysiologie der Universitätsmedizin Göttingen die kortikale Erregbarkeit während des Sprechens und der Sprechplanung gemessen.

Das Ziel dieser Studie war, die kortikale Erregbarkeit in der Übergangsphase zwischen zwei Sprechbewegungen bei stotternden Personen und der Kontrollgruppe zu ermitteln. Es ist gelungen, die Modulation der Erregbarkeit des Motorkortex während der Sprechaufgaben und zwar während der motorischen Sprechplanung und der zeitgesteuerten Initiierung der sensomotorischen Sprechprogramme aufzuzeichnen. Es konnten in dieser Studie Daten über (1) die motorische Kortex-Erregbarkeit während des willkürlichen Ausdehnens des Präfixes "auf' und (2) während der Übergangsphase zwischen dem gleichbleibenden Präfix "auf" und der anschließenden Artikulation eines variablen Verbs gewonnen werden. Das Hauptergebnis der Studie war, dass ein Anstieg der Erregbarkeit, insbesondere der kontralateralen Zungenseite bei der Stimulation des linken Motorkortex der Zungenrepräsentation mittels der TMS bei flüssig sprechenden Probanden vor dem zweiten Sprechsegment, also während der Vorbereitungsphase der zweiten Sprechbewegung aufgezeichnet wurde. 
Wohingegen auf dem rechten Motorkortex der Zungenrepräsentation in dieser Phase weniger Erregung nachgewiesen wurde. Somit weisen flüssig sprechende Probanden eine spezifische Fazilitation im linken primären Motorkortex auf, während die Erregbarkeit im rechten primären Motorkortex nicht moduliert wird. Dieses Ergebnis wird durch das Sprachmodell auf der Basis funktioneller Bildgebung bestätigt (Hickok und Poeppel 2007). Dieser Anstieg der MEPAmplituden auf dem linken Motorkortex während der Übergangsphase zwischen zwei Sprechsegmenten konnte dagegen bei stotternden Personen nicht festgestellt werden. Zusätzlich zeigten stotternde Erwachsenen eine Tendenz zur Fazilitation auf dem rechten primären Motorkortex. Dieses Ergebnis ist gut mit den bildgebenden Studien von Braun et al. (1997) und Fox et al. (1996) vereinbar. In diesen Studien wurde bei stotternden Erwachsenen eine erhöhte Aktivität auf dem rechten Motorkortex und eine Unteraktivität des linken Motorkortex beschrieben.

Diese Ergebnisse deuten auf eine gestörte Vorbereitung oder ein verändertes Vorbereitungsmuster im linken primären Motorkortex während der Übergangsphase zwischen zwei artikulatorischen Sprechbewegungen als einen plausiblen pathophysiologischen Mechanismus beim Stottern hin. Es erklärt auch die klinisch ausgeprägte Unfähigkeit stotternder Erwachsener von einer Sprechsequenz auf die anschließende artikulatorische Bewegung flüssig überzugehen (Wingate 2002). Zusätzlich wird die kritische pathophysiologische Eigenschaft des linken primären Motorkortex durch die negative Korrelation der Stotterfrequenz und der Fazilitation zwischen den zwei Sprechbewegungen verdeutlicht (siehe Abbildung 7).

Kornhuber und Deecke (1965) entdeckten die Gehirnaktivität unmittelbar vor einer Willkürbewegung und bezeichneten diese als "Bereitschaftspotential", welches als Grundlage zahlreicher Untersuchungen der Willkürbewegung diente. Im Jahre 1986 untersuchten Deecke et al. das bilaterale Bereitschaftspotential zwei Sekunden vor einem Sprechbeginn und fanden eine signifikante Lateralisation in die linke Hemisphäre in den letzten 100 ms vor dem Beginn des Sprechens bei stotternden Personen. Sie bezeichneten die $200 \mathrm{~ms}$ vor dem Sprechbeginn als die Vorbereitungszeit, die bei stotternden Personen nicht vorhanden war. Diese Gehirnaktivität ist für den Motorantrieb der Sprechproduktion erforderlich.

Walla et al. (2004) aus der Arbeitsgruppe von Deecke untersuchten mittels Magnetenzephalographie (MEG) die zerebrale Aktivität vor der Sprechbewegung. 
Sie zeigten, dass bei stotternden Personen die Gehirnaktivität während der Vorbereitung auf die Einzel-Wort-Sprechaufgabe fehlt und interpretierten dies als Beeinträchtigung der fokussierten Aufmerksamkeit oder der Antizipation der verbalen Informationsverarbeitung. In einer anderen Sprechaufgabe konnte die Gehirnaktivität der artikulatorischen Planung und motorischen Vorbereitung bei flüssig sprechenden Probanden nachgewiesen werden, die bei stotternden Personen nicht vorhanden war (Walter 1964).

Die Daten dieser Studie zeigen eine Asymmetrie der Kortexerregbarkeit bei stotternden Personen zwischen zwei Sprechbewegungen. Die Frage ist, was den Grund für diese Fehlverbindung darstellt. Weiterführende Forschungsansätze zur Erklärung der Fehlverbindungen der sprachrelevanten Hirnareale erfolgten. Mittels Diffusion Tensor Imaging und Fraktionaler Anisotropie untersuchten Sommer et al. (2002) beispielsweise die Stärke der weißen Substanz unmittelbar unterhalb der Repräsentation des Kehlkopfes und der Zunge im sensomotorischen Kortex und fanden kortikale Fehlverbindung auf dem linken sensomotorischen Kortex bei stotternden Personen. Des Weiteren wurden strukturelle Abnormitäten der sprachrelevanten Hirnareale auf dem linken Rolandischen Operculum bei stotternden Personen beobachtet (Sommer 2002; Cykowski et al. 2010).

Zusätzlich ist der Pathomechanismus einer Funktionsstörung im linken Motorkortex zu diskutieren. Aktuelle Studien liefern im Bezug auf strukturelle und funktionelle Defizite der linken inferioren frontalen prämotorischen Verbindungen bei stotternden Personen Hinweise auf eine Lateralisierungstherorie. Das Ergebnis dieser Untersuchungen ergab als neuronales Korrelat bei stotternden Personen eine unzureichende Verbindung vom linken inferioren frontalen Kortex zum Prämotorkortex (Chang et al. 2011). Einer der zwei wichtigsten Schlüsselpunkte dieser Studie war die Fehlverbindung der kortikokortikalen Verknüpfung der artikulatorischen Region innerhalb des frontalen motorischen Areals mit den auditorisch-sensorischen Arealen im temporoparietalen Kortex und führte somit zur funktionellen und kortikalen Verbindungsdifferenz auf der linken Hemisphäre. Der zweite Schlüsselpunkt der Studie beschrieb die funktionelle Verbindungsdifferenz in den thalamokortikalen Bahnen zwischen stotternden Personen und der Kontrollgruppe. Es konnte eine signifikant stärkere funktionelle Verbindung zwischen dem linken BA44 und den bilateralen sprachrelevanten Hirnregionen bei flüssig sprechenden Probanden als bei stotternden Personen 
gezeigt werden. Stattdessen wurden bei stotternden Personen verstärkte Verbindungen auf der rechten Hemisphäre mit den bilateralen sprachrelevanten Hirnarealen beobachtet (Chang et al. 2011).

In Anbetracht der strukturellen und funktionellen Abnormitäten des motorischen Systems bei stotternden Personen unterstützen die Ergebnisse dieser Studie die bisherigen Untersuchungen von Watkins et al. (2008). Sie fanden ebenfalls eine Unteraktivität im ventralen Prämotorkortex, im Rolandischem Operculum, im bilateralen sensomotorischen Kortex und im Hensch`s Gyrus auf der linken Hemisphäre, welche mit der Artikulation und Sprachproduktion assoziiert sind.

Dass die stotternden Personen in dieser Studie keine Fazilitation in der Übergangsphase zeigten, könnte auf eine abgeschwächte und veränderte strukturelle Verbindung zwischen den linkshemisphärischen sprachrelevanten Hirnregionen hindeuten. Dabei wird der Funktion der Integration der weißen Substanz eine große Bedeutung beigemessen. Dieses Fehlverbindungsproblem beim Stottern wird durch zahlreiche Untersuchungen untermauert (Sommer 2002; Chang et al. 2008; Watkins et al. 2008; Cykowski et al. 2010; Cai et al. 2014).

Die Frage nach Ursache oder Folge (Henne oder Ei) solcher hirnstruktureller Veränderungen ist durch Untersuchungen seit der Kindheit stotternder Erwachsener nur eingeschränkt zu beantworten. Hierzu sind Studien an Kindern mit beginnendem Stottern erforderlich. Chang et al. (2008) verglichen das Volumen der grauen Substanz und die Tensor-Diffusion in den Bahnen der weißen Substanz bei stotternden Kindern, bei Kindern, die eine Remission des Stotterns erfahren haben, und bei flüssig sprechenden Kindern. Bei den stotternden Kindern und Kindern mit Remission konnten reduzierte Volumina der grauen Substanz in den sprachrelevanten Hirnregionen, d. h. im linken GFI und in den bilateralen Temporalregionen festgestellt werden.

Eine reduzierte fraktionale Anisotropie auf den linken Bahnen der weißen Substanz unterhalb der Repräsentation des Gesichts und des Larynx wurde bei stotternden Kindern als auch bei stotternden Kindern mit Remission gefunden, jedoch ohne einen Anstieg auf der rechten Hemisphäre der Sprachregion.

Der Anstieg der Erregbarkeit der weißen Substanz auf der rechten Hemisphäre bei stotternden Erwachsenen spricht deshalb eher für eine Kompensation der Dysfunktion auf der linken Hemisphäre bei CIS (Chang et al. 2008). In der Tat 
zeigten stotternde Personen im Vergleich zu flüssig sprechenden Probanden ein signifikant größeres Volumen der weißen Substanz, welche eine Verbindung des linken Motorkortex mit den linken superioren longitudinalen Faszikeln darstellt. Eine solche Verbindungsstörung weist auf ein Defizit der Übertragung der sensomotorischen Sprechprogramme vom linken BA44/B6 in den M1 der Zungenrepräsentation hin (Chang et al. 2008).

Durch diese sogenannte kortikokortikale Verbindung kann die motorische Planung der sensomotorischen Sprechprogramme im GFI Einfluss auf die Kortexerregbarkeit ausüben (Greenlee et al. 2004).

Ein reduziertes Volumen der weißen Substanz kann an anderer Stelle die kortikostriatale Verbindung verhindern und dadurch die zeitliche Koordinierung der anschließenden Sprechsegmente über die kortikothalamokortikale Schleife unterbinden (Alm 2004).

Eine intakte Myelinisierung ist notwendig, damit die Funktion der weißen Substanz, nämlich der Transport der Information von der grauen Substanz zur peripheren Muskulatur gewährleistet ist. Hier kann nur spekuliert werden, ob es sich um deszensierende Bahnen handelt oder vielmehr um Assoziationsfasern im Gehirn selber. Also etwa Verbindungsbahnen zwischen motorischer Hirnrinde und dem Broca-Areal. Die normale Rekrutierung spricht jedoch eher dafür, dass Assoziationsfasern betroffen sind. Das steht im Einklang mit Cykowski et al. (2010). Die Autoren untersuchten die Qualität der afferenten Nerven und fanden eine eingeschränkte Myelinisierung bei stotternden Erwachsenen innerhalb der linken hemisphärischen Nervenbahnen. Die Relevanz dieser afferenten Nerven für die Sprachproduktion wurde mittels der Fraktionalen Anisotropie bei stotternden Erwachsenen (Sommer 2002) und stotternden Kindern (Chang et al. 2008) nachgewiesen.

Unter Anbetracht der kognitiven Prozesse während der Sprechaufgaben können die komplexen Wechselwirkungen der beteiligten Hirnareale anhand des GODIVASprachmodells (Gradient Order Directions into Velocities of Articulators) erklärt werden (Bohland et al. 2010). In diesem Modell sind zwei unabhängige Mechanismen im M1 als ausführende Prozesse der kortikalen und subkortikalen Kontrolle der flüssigen Sprachproduktion beschrieben worden. Es werden dabei die motorischen Output-Zellen (Ausführungszellen) von den motorischen Planzellen unterschieden. Während die motorischen Output-Zellen das Timing und 
die Initiierung des sensomotorischen Programms koordinieren, wird den motorischen Planzellen die Funktion der Phonation und Vokalisation zugeschrieben. Es wird angenommen, dass die motorischen Planzellen nur im linken orofazialen Kortex lokalisiert sind, während die motorischen Ausführungszellen sowohl im linken als auch im rechten Motorkortex zu finden sind (Terumitsu et al. 2006). Eine große Rolle bei der Freisetzung der geplanten Sprachlaute spielt v.a. die Interaktion des prä-SMA mit dem Putamen, dem Globus pallidum und dem Thalamus. Dabei ist beidseits der Motorkortex involviert. Die Entkopplung der motorischen Output-Zellen von den motorischen Planzellen ermöglicht die Vorbereitung einer Sprechsequenz. Dabei wird das sensomotorische Sprachprogramm auf eine Sprechsequenz vorbereitet, während der vorherige Sprechakt ausgeführt wird (Bohland et al. 2010). In dieser Studie wurde die Erregbarkeit des linken orofazialen Motorkortex vor der zweiten Sprechbewegung bei flüssig sprechenden Probanden temporär erhöht. Dieses Ergebnis deutet auf solch eine Entkopplung der sprechmotorischen Planung im linken Motorkortex von der Initiierung der Sprachproduktion im Motorkortex beidseits hin. Folglich kann angenommen werden, dass die vorübergehende Erhöhung der Kortexerregbarkeit vor der zweiten Sprechbewegung auf das Warten auf die Initiierung des motorischen Sprechprogramms hindeutet.

Ein unausgewogener Dopaminspiegel kann die kortikale Erregbarkeit modulieren. Bei stotternden Personen werden die Symptome durch Dopaminantagonisten gedämpft. Auch bei gesunden Probanden konnte man beobachten, dass die intrakortikale Erregbarkeit durch die Gabe von Dopaminagonisten Bromocriptin und Cabergolin reduziert wurden (Wu et al. 1997; Ziemann et al. 1997; Korchounov et al. 2007). 


\section{Fazit}

In dieser Arbeit wurden die Probanden gebeten den Reibelaut des deutschen Präfixes "auf" willkürlich zu verlängern (Prolongation). Für flüssig sprechende Probanden war diese Studienbedingung eine künstliche, während sie für stotternde Personen teilweise unwillkürlich passiert. Wir verglichen Werte der Studienergebnisse während der Ruhephase, Werte während der Prolongation des Präfix "auf" und Werte zwischen den zwei Sprechbewegungen.

In der Ruhephase zeigten flüssig sprechende Probanden eine höhere Exzitabilität sowohl bei der Stimulation des linken als auch des rechten Motorkortex. In der kontralateralen Zungenrepräsentation ist die Exzitabilität am deutlichsten zu erkennen.

Während der Prolongation des Präfix "auf" zeigten stotternde Erwachsenen eine erhöhte Exzitabilität im linken orofazialen Motorkortex, insbesondere auf der kontralateralen Zungenprojektion. Bei flüssig sprechenden Probanden konnte dagegen eine Tendenz zur erhöhten Exzitabilität bei der Stimulation des rechten Motorkortex verzeichnet werden. Die Zeit während der Prolongation des Präfixes spiegelt die Vorbereitung für die Herstellung des nächsten Wortes nach dem zweiten Go-Signal wider. Die erhöhte Exzitabiltät während der Prolongation des Präfix bei stotternden Personen kann ein verändertes Vorbereitungsverhalten mit einer übermäßigen Aktivierung des M1 bedeuten (Salmelin et al. 2000).

Während der Übergangsphase, der Phase zwischen der Prolongation des Präfixes "auf" und vor der zweiten artikulatorischen Bewegung, ist bei flüssig sprechenden Probanden eine links-lateralisierte Erhöhung der Motorkortexerregbarkeit gemessen worden. Diese Erhöhung war bei stotternden Erwachsenen nicht nachzuweisen, dagegen zeigten sie eine rechts-lateralisierte Erhöhung der Kortexerregbarkeit. Dieses Ergebnis deutet auf eine abgeschwächte und veränderte strukturelle Verbindung zwischen den linkshemisphärischen sprachrelevanten Hirnregionen hin.

Bisherige Studien zur Fehlfunktion des linken Motorkortex und Fehlverbindungen innerhalb der sprachrelevanten Regionen konnten die Frage nach einer Kompensation nicht abschließend beantworten. Bildgebende Studien von Fox et al. (1996) und Braun et al. (1997) beschrieben lediglich die hohe Aktivität des 
rechten Motorkortex und die Unteraktivität des linken Motorkortex bei stotternden Personen. Das Ergebnis dieser Studie kann die Kompensation der rechten Hemisphäre bei ansteigenden MEP-Amplituden nach der Stimulation des linken Motorkortex nicht zeigen. Die Daten deuten jedoch auf eine Tendenz des Anstiegs der MEP-Amplituden nach der Stimulation des rechten Motorkortex bei stotternden Erwachsenen hin. Wenn überhaupt eine Exzitabilitätsmodulation bei stotternden Erwachsenen vorliegt, dann ist diese nach den Studienergebnissen auf der rechten Hemisphäre zu finden.

Die Daten binden strukturelle und neurophysiologische Erkenntnisse in ein plausibles Modell der Sprechpathophysiologie bei stotternden Personen ein. In diesem Modell wurde die zeitlich fehlerhafte Interaktion des GFI mit dem Motorareal (Salmelin et al. 2000) und ein Mangel der funktionellen Verbindung zwischen dem linken GFI und dem linken M1 (Chang et al. 2011) unterstützt. Im Rahmen des "hierarchischen Zustandsregelungsmodells" (Hickok 2012) erlauben die Ergebnisse der Arbeit, die Pathophysiologie des chronischen Stotterns zwischen dem Broca-Areal und dem linken M1, d. h. am Übergang von der Silbe zur artikulatorischen Wortzusammensetzung, zu lokalisieren.

Darüber hinaus unterstützen die Ergebnisse das theoretische Konzept der sprechmotorischen Kontrolle, nämlich des GODIVA-Sprachmodells (Bohland et al. 2010). Im Hinblick auf dieses Modell ergaben die Daten, dass das Stottern mit einer unzureichenden Aktivierung der sensomotorischen Programme im linken orofazialen Motorkortex verbunden war, da für die segmentale und suprasegmentale Sprechplanung eine intakte kortikokortikale Verbindung vom BA44/BA6 zum supplementären Areal erforderlich ist.

Die Daten dieser Untersuchung deuten darauf hin, dass die Steigerung der Erregbarkeit im sprachspezifischen linken motorischen Kortex bei Flüssigsprechenden vorhanden ist, während diese bei stotternden Erwachsenen fehlt. Darüber hinaus konnte ein Zusammenhang zwischen dem Stotternschweregrad und der Erregbarkeit bei stotternden Personen festgestellt werden (Neef et al. 2015).

Die vorliegende Arbeit befasst sich mit fundamentalen Änderungen der Sprechvorbereitung stotternder Menschen. Sie kann aber nicht erklären, warum trotz der gefundenen Auffälligkeiten große Anteile des Sprechflusses stotternder Menschen flüssig sind und was die gestotterten Sprechabschnitte von den 
flüssigen unterscheidet. Hierzu ist eine genaue Differenzierung gestotterter und nicht gestotterter Sprechabschnitte in Bezug auf die kortikale Bewegungsvorbereitung notwendig. Weitere Untersuchungen sind essentiell. 


\title{
7. Anhang
}

Anhang 1 - Informationsblatt für Probanden

Studienstelle: Abteilung Klinische Neurophysiologie, Medizinische Fakultät, Universität Göttingen, Robert-Koch-Str. 40, 37070 Göttingen, Tel.: 0551/396650, Telefax: 0551/398126

Studienarzt: Prof. Dr. med. Martin Sommer, E-Mail: msommer@gwdg.de

Titel der Studie

\section{Sprechbezogene Veränderungen der Erregbarkeit des primären Motorkortex bei Stotternden und Kontrollprobanden}

\author{
Untersuchungsteil: TMS
}

Sehr geehrte Probandin, sehr geehrter Proband,

wir möchten Sie fragen, ob Sie bereit sind, an der nachfolgend beschriebenen wissenschaftlichen Studie teilzunehmen.

Wissenschaftliche Studien sind notwendig, um Erkenntnisse über neurobiologische Zusammenhänge zu gewinnen und wissenschaftlich fundierte Therapiemethoden zu entwickeln und zu verbessern. Die wissenschaftliche Studie, die wir Ihnen hier vorstellen, wurde - wie es das Gesetz verlangt - von der zuständigen Ethikkommission zustimmend bewertet. Diese Studie wird in der Abteilung Klinische Neurophysiologie, Göttingen durchgeführt; es sollen insgesamt ungefähr 20 stotternde und 20 nicht stotternde Personen daran teilnehmen. Die Studie wird veranlasst, organisiert und finanziert durch die Universitätsmedizin Göttingen, dem Sponsor dieser Studie.

Ihre Teilnahme an dieser wissenschaftlichen Studie ist freiwillig. Sie werden in diese Studie also nur dann einbezogen, wenn Sie dazu schriftlich Ihre Einwilligung erklären. Sofern Sie nicht an der wissenschaftlichen Studie teilnehmen oder später aus ihr ausscheiden möchten, erwachsen Ihnen daraus keine Nachteile.

Sie wurden bereits auf die geplante Studie angesprochen. Der nachfolgende Text soll Ihnen die Ziele und den Ablauf erläutern. Anschließend wird ein Studiendurchführender das Aufklärungsgespräch mit Ihnen führen. Bitte zögern Sie nicht, alle Punkte anzusprechen, die Ihnen unklar sind. Sie werden danach ausreichend Bedenkzeit erhalten, um über Ihre Teilnahme zu entscheiden.

\section{Warum wird diese Studie durchgeführt?}

Die Ursache des in der Kindheit entstehenden Stotterns ist weitgehend unbekannt. Wir wollen daher beobachten, was das Gehirn beim Sprechen tut. Dazu wollen wir Erregungs- und Hemmungsvorgänge in den für die Sprechbewegung zuständigen 
Bereiche des Gehirns messen, und zwar mit Hilfe von durch die Kopfhaut und den Schädel (transkraniell) gegebenen Magnetreizen.

Diese Untersuchung wird dazu beitragen, dem Stottern zugrundeliegende Störungen in den für die Sprechbewegungen zuständigen Bereichen des Gehirn besser zu verstehen.

\section{Wie ist der Ablauf der Studie und was muss ich bei Teilnahme beachten?}

Vorbereitung: Zunächst wollen wir Sie über die Studie aufklären. Nach Ihrer Einwilligung werden wir einige Fragen zu Ihrer Person stellen und zur Beurteilung der Sprechflüssigkeit eine Sprechprobe mit Video aufzeichnen. Hierfür bitten wir Sie einen Text vorzulesen und so ausführlich wie möglich über ihren letzten Arbeitstag/Studientag und über einen Film/Buch zu berichten und eine Wegbeschreibung zu geben. Auf diesem Video werden Sie vollständig zu sehen sein.

Dann wollen wir die Erregbarkeit der für die Zunge zuständigen Gehirnzellen untersuchen. Dazu wollen wir die sogenannte transkranielle Magnetstimulation verwenden. Dies Untersuchungsmethode ist schmerzfrei und kommt ohne Verletzung der Haut aus. Die Stimulation erfolgt mittels einer Reizspule, die auf Ihren Kopf aufgelegt wird. Bei überschwelliger Stimulation werden Sie eine leichte unwillkürliche Zuckung in einigen Muskeln Zunge verspüren. Dazu legen wir einen sterilisierten Speziallöffel auf Ihre Zunge; auf diesen Löffel kleben wir OberflächenEinmalelektroden, welche diese Zuckungen registrieren.

Diese Untersuchungen finden zunächst in Ruhe statt. Anschließend wollen wir diese Untersuchungen mehrfach wiederholen, während Sie sprechen. Dazu werden wir Sie bitten, sich auf einem Bildschirm gezeigte Worte zu merken und auf ein Signal hin auszusprechen. Diese Sprechaufgabe wollen wir mehrfach mit unterschiedlichen Worten wiederholen.

Insgesamt erhalten Sie in bei diesen Untersuchungen ca. 1000 Magnetreize, der Abstand zwischen zwei Einzelreizen oder zwei Reizpaaren beträgt wenigstens 4 Sekunde.

Die gesamte Untersuchung dauert inklusive Voruntersuchungen vier bis fünf Stunden, was auf zwei Termine aufgeteilt werden kann.

Aus den durchgeführten Untersuchungen, die gelegentlich medizinisch bedeutsame Tatsachen zu Tage fördern können, kann keinerlei Anspruch auf eine vollständige Gesundheitsuntersuchung abgeleitet werden.

Bitte fragen Sie uns, wenn Sie etwas nicht verstanden haben oder wenn Sie mehr über die Untersuchungsmethode erfahren wollen. Wir geben Ihnen gerne weitere Auskunft im Gespräch. Bitte fragen Sie nach allem, was Ihnen wichtig erscheint.

\section{Welchen persönlichen Nutzen habe ich von der Teilnahme an der Studie?}

Sie werden durch die Teilnahme an dieser Studie keinen persönlichen Gesundheitsnutzen haben. Die Ergebnisse der Studie können aber 
möglicherweise dazu beitragen, die Diagnose und Behandlung von persistentem Entwicklungsstottern zukünftig zu verbessern bzw. besser beurteilen zu können.

\section{Welche Risiken sind mit der Teilnahme an der Studie verbunden?}

Wegeunfälle zur und von der Untersuchungsstelle sind nicht versichert.

Die Untersuchungsmethode der transkraniellen Magnetstimulation ist schmerzfrei und kommt ohne Verletzung der Haut aus. Die bereits vorliegenden, umfangreichen Erfahrungen haben gezeigt, daß dieses Verfahren risiko- und nebenwirkungsarm ist, wenn die Ausschlußkriterien beachtet werden (siehe unten). In seltenen Fällen ist mit Auftreten von Müdigkeit und Kopfschmerzen zu rechnen. Die TMS kann einen epileptischen Anfall auslösen. Bei der Verwendung einzelner Pulse ist jedoch das Risiko einen Anfall auszulösen äußerst gering. In unserem Labor wurde noch nie ein epileptischer Anfall mittels TMS ausgelöst.

\section{Wer darf an dieser wissenschaftlichen Studie nicht teilnehmen?}

Falls einer der folgenden Punkte auf Sie zutrifft, ist eine Teilnahme an der Studie nicht möglich:

1. Andere neurologische Erkrankungen, akut oder in der Vorgeschichte (z. B. Epilepsie, Hirntumor)

2. Alter $<18$ oder $>70$.

3. Metallimplantate im Kopf oder Herzschrittmacher, metallenthaltene Objekte / andere Objekte mit Risiken bei Kontakt mit den Elektroden.

4. Vorliegen einer gravierenden internistischen (Erkrankung der inneren Organe: schwere Hypertonie, dekompensierte Herzinsuffizienz) oder psychiatrischen (seelische Erkrankung) Vorerkrankung, insbesondere Schizophrenie (seelische Erkrankung mit Wahn, Trugwahrnehmungen und Störungen des Denkens) oder Manie (Erkrankung des Gemütes mit gehobener Stimmung).

5. Drogen-, Medikamenten- und/oder Alkoholabhängigkeit

6. Rezeptive oder globale Aphasie (Störung des Sprachverständnisses bzw. zusätzlich des Sprechens)

7. Hörstörung

8. gleichzeitige Teilnahme an einer anderen wissenschaftlichen oder klinischen Studie.

\section{Entstehen für mich Kosten durch die Teilnahme an der wissenschaftlichen Studie? Erhalte ich eine Aufwandsentschädigung?}

Durch die Teilnahme an dieser Studie entstehen für Sie keine zusätzlichen Kosten. Für Ihre Teilnahme an dieser wissenschaftlichen Studie erhalten Sie eine Aufwandsentschädigung von 7,00 EUR pro Stunde. 


\section{Werden mir neue Erkenntnisse während der wissenschaftlichen Studie mitgeteilt?}

Sie werden über neue Erkenntnisse, die in Bezug auf diese wissenschaftliche Studie bekannt werden, informiert.

\section{Kann meine Teilnahme an der wissenschaftlichen Studie vorzeitig beendet werden?}

Sie können jederzeit, auch ohne Angabe von Gründen, Ihre Teilnahme beenden. Unter gewissen Umständen ist es aber auch möglich, dass die Studiendurchführenden Ihre Teilnahme an der wissenschaftlichen Studie vorzeitig zu beenden, ohne dass Sie auf die Entscheidung Einfluss haben. Die Gründe hierfür können z. B. sein, dass die gesamte wissenschaftliche Studie abgebrochen wird.

\section{Was geschieht mit meinen Daten?}

Während der Studie werden personenbezogene Daten erhoben und nur in pseudonymisierter Form aufgezeichnet. Pseudonymisierung bedeutet Verschlüsselung von Daten/Proben ohne Namensnennung nur mit Nummern codiert. Die Zuordnung der Daten oder Proben zu einer Person ist nur möglich, wenn hierfür der Schlüssel eingesetzt wird, mit dem die Daten pseudonymisiert wurden. Die personenbezogenen Daten/Proben werden unter besonderen Schutzvorkehrungen getrennt von der Schlüsselliste aufbewahrt. Eine Entschlüsselung ist nur durch die verantwortlichen Studiendurchführenden möglich. Dritte erhalten keinen Einblick in Originalunterlagen.

Auf den Videos zur Erfassung der Sprechflüssigkeit werden Sie vollständig zu sehen sein. Die angefertigten Videos werden pseudonymisiert in der Abteilung Klinische Neurophysiologie aufbewahrt, das heißt der Videodatei wird ein Buchstabencode zugeordnet, der nur von der Studienleiterin Nicole Neef entschlüsselt werden kann und das Video wird ausschließlich von Nicole Neef zur Analyse eingesehen. Das Videomaterial wird nicht vorgeführt sondern ausschließlich im Rahmen der Studie genutzt. Restmaterial wird bei Abschluss der Studie vernichtet. Bei Widerruf der Studienteilnahme werden alle Videodaten gelöscht.

Die gesetzlichen Bestimmungen enthalten nähere Vorgaben für den erforderlichen Umfang der Einwilligung in die Datenerhebung und -verwendung. Die Verwendung der Angaben über Ihre Gesundheit erfolgt nach gesetzlichen Bestimmungen und setzt vor der Teilnahme an der wissenschaftlichen Studie eine freiwillig 
abgegebene Einwilligungserklärung voraus, das heißt ohne die nachfolgende Einwilligung können Sie nicht an der wissenschaftlichen Studie teilnehmen. Sie können jederzeit die Teilnahme an der wissenschaftlichen Studie beenden. Beim Widerruf Ihrer Einwilligung, an der Studie teilzunehmen, werden alle bis dahin gespeicherten personenbezogenen Daten gelöscht.

Ihre Daten werden nach Beendigung oder Abbruch der Studie mindestens zehn Jahre aufbewahrt. Danach werden Ihre personenbezogenen Daten gelöscht.

Die ärztliche Schweigepflicht und die Bestimmungen der Datenschutzgesetze werden eingehalten.

Bei der Veröffentlichung von Ergebnissen der Studie wird der Name ebenfalls nicht genannt.

\section{An wen wende ich mich bei weiteren Fragen?}

\section{Beratungsgespräche an der Studienstelle}

Sie haben stets die Gelegenheit zu weiteren Beratungsgesprächen mit dem auf Seite 1 genannten oder einem anderen Studiendurchführenden.

\begin{tabular}{|l|l|}
\hline $\begin{array}{l}\text { Studiendurchführende: } \\
\text { Apl Prof. Dr. Martin Sommer }\end{array}$ & Dr. rer. nat. Dipl. Lehrlogopädin N. \\
Abteilung Klinische Neurophysiologie & $\begin{array}{l}\text { Abteilung Klinische Neurophysiologie } \\
\text { Georg-August-Universität Göttingen }\end{array}$ \\
UMG & UMG \\
Robert Koch Str. 40 & Robert Koch Str. 40 \\
$\begin{array}{l}\text { G7075 Göttingen } \\
\text { Tel.: 0551/398463 }\end{array}$ & 37075 Göttingen \\
\hline Assistenzärztin Thi Ngoc Linh Hoang & \\
Abteilung Klinische Neurophysiologie & \\
Georg-August-Universität Göttingen & \\
UMG & \\
Robert Koch Str. 40 & \\
37075 Göttingen & \\
Tel.: 0551/3912631 & \\
\hline
\end{tabular}




\section{Anhang 2 - Einverständniserklärung für Probanden}

Studienstelle: Abteilung Klinische Neurophysiologie, Medizinische Fakultät, Universität Göttingen, Robert-Koch-Str. 40, 37070 Göttingen, Tel.: 0551/396650, Telefax: 0551/398126

Studienarzt: Prof. Dr. med. Martin Sommer, E-Mail: msommer@gwdg.de

\section{Titel der Studie}

\section{Sprechbezogene Veränderungen der Erregbarkeit des primären Motorkortex bei Stotternden und Kontrollprobanden}

Untersuchungsteil: TMS

\section{Einwilligungserklärung}

Name des Probanden/ der Probandin in Druckbuchstaben

Ich bin in einem persönlichen Gespräch durch den Studiendurchführenden/ die Studiendurchführende

Name der Studiendurchführenden/ des Studiendurchführenden

ausführlich und verständlich über Wesen, Bedeutung, Risiken und Tragweite der wissenschaftlichen Studie aufgeklärt worden. Ich habe darüber hinaus den Text der Teilnehmerinformation sowie die hier nachfolgend abgedruckte Datenschutzerklärung gelesen und verstanden. Ich hatte die Gelegenheit, mit dem Studiendurchführenden über die Durchführung der wissenschaftlichen Studie zu sprechen. Alle meine Fragen wurden zufrieden stellend beantwortet.

Möglichkeit zur Dokumentation zusätzlicher Fragen seitens des Teilnehmers oder sonstiger Aspekte des Aufklärungsgesprächs: 
Ich hatte ausreichend Zeit, mich zu entscheiden.

Mir ist bekannt, dass ich jederzeit und ohne Angabe von Gründen meine Einwilligung zur Teilnahme an der Studie zurückziehen kann (mündlich oder schriftlich), ohne dass mir daraus Nachteile für meine medizinische Behandlung entstehen.

\section{Datenschutz:}

Mir ist bekannt, dass im Rahmen dieses Forschungsvorhabens personenbezogenen Daten erhoben und in pseudonymisierter (verschlüsselter) Form in aufgezeichnet und gespeichert werden. Die Datenspeicherzeit beträgt 10 Jahre. Die personenbezogenen Daten werden nicht an Dritte weitergegeben. Ich weiß, dass ich mein Einverständnis zur Speicherung der personenbezogenen Daten jederzeit widerrufen kann. Im Falle des Widerrufs werden alle personenbezogenen Daten gelöscht.

Ich erkläre mich damit einverstanden, dass eine Videoaufnahme, auf der ich vollständig zu sehen bin, psydonymisiert gespeichert werden und von Frau Nicole Neef eingesehen werden darf. Das Video wird 10 Jahre lang gespeichert. Bei Widerruf wird das Video gelöscht. 


\section{Ich erkläre mich bereit, an der oben genannten wissenschaftlichen Studie freiwillig teilzunehmen.}

Ein Exemplar der Probanden-Information und -Einwilligung habe ich erhalten. Ein Exemplar verbleibt im Studienzentrum.

Name des Probanden/ der Probandin in Druckbuchstaben

Datum

Unterschrift

des

Probanden/

der

Probandin

Ich habe das Aufklärungsgespräch geführt und die Einwilligung des Probanden/ der Probandin eingeholt.

Name des Studiendurchführenden / der Studiendurchführenden in Druckbuchstaben

Datum

Unterschrift des aufklärenden Studiendurchführenden/

der

Studiendurchführenden 


\section{Anhang 3 - Händigkeitsfragebogen - Edingburgh Handedness Inventory (nach Oldfield 1971)}

Probandenkürzel:

Datum:

○ nicht durchgeführt Grund:

Bitte kreuzen Sie an, mit welcher Hand Sie vorzugsweise die unten genannten Tätigkeiten ausführen. Falls der Vorzug so stark ist, dass Sie niemals die andere Hand benutzen würden, wenn Sie nicht dazu gezwungen wären, machen Sie bitte zwei Kreuze in dem entsprechenden Feld. Falls Sie nicht für eine Hand entscheiden können, kreuzen Sie bitte beide Möglichkeiten an. Einige der Tätigkeiten, erfordern beide Hände. In diesem Fall ist der Teil der Tätigkeit bzw. des Objekts, dessen Händigkeit gefragt ist, in Klammern angegeben. Bitte versuchen Sie alle Fragen zu beantworten. Falls Sie keine Erfahrung mit der genannten Tätigkeit haben, lassen Sie entsprechende Zeile frei.

\begin{tabular}{|l|l|l|l|}
\hline \multicolumn{2}{|l|}{} & links & rechts \\
\hline 1. & Schreiben & & \\
\hline 2. & Zeichnen & & \\
\hline 3. & Werfen & & \\
\hline 4. & Schere & & \\
\hline 5. & Zahnbürste & & \\
\hline 6. & Messer (ohne Gabel) & & \\
\hline 7. & Löffel & & \\
\hline 8. & Besen (obere Hand) & & \\
\hline 9. & Streichholz anzünden (Streichholz) & & \\
\hline 10. & Schachtel öffnen (Deckel) & & \\
\hline
\end{tabular}




\section{Literaturverzeichnis}

Ackermann H, Riecker A (2004): The contribution of the insula to motor aspects of speech production: A review and a hypothesis. Brain Lang 89 , 320-328

Alm PA (2004): Stuttering and the basal ganglia circuits: a critical review of possible relations. J Commun Disord 37, 325-369

Ambrose NG, Cox NJ, Yairi E (1997): The genetic basis of persistence and recovery in stuttering. J Speech Lang Hear Res 40, 567-580

Andrews G: Epidemiology of stuttering. In: Curlee RF, Siegel GM (Hrsg.): Nature and Treatment of Stuttering: New Directions. College-Hill Press, San Diego 1985

Andrews G, Harris M: The syndrome of stuttering. Clinics in developmental medicine 17. William Heinemann Medical Books, London 1964

Andrews G, Quinn PT, Sorby WA (1972): Stuttering: an investigation into cerebral dominance for speech. J Neurol Neurosurg Psychiatry 35, 414-418

Andrews G, Craig A, Feyer AM, Hoddinott S, Howie P, Neilson M (1983): Stuttering: a review of research findings and theories circa 1982. J Speech Hear Disord 48, 226-246

Andrews G, Morris-Yates A, Howie P, Martin NG (1991): Genetic factors in stuttering confirmed. Arch Gen Psychiatry 48, 1034-1035

Barker AT, Jalinous R, Freeston IL (1985): Non-invasive magnetic stimulation of human motor cortex. Lancet 325, 1106-1107

Benninghoff A, Fleischhauer K: Makroskopische und mikroskopische Anatomie des Menschen 2. Band. Urban \& Schwarzenberg, München 1985

Bloodstein O: A handbook on stuttering. 5. Auflage; Singular Pub. Group, San Diego 1995

Blumgart E, Tran Y, Craig A (2010): An investigation into the personal financial costs associated with stuttering. J Fluen Disord 35, 203-215

Bohland JW, Guenther FH (2006): An fMRI investigation of syllable sequence production. Neurolmage 32, 821-841

Bohland JW, Bullock D, Guenther FG (2010): Neural representations and mechanisms for the performance of simple speech sequences. J Cogn Neurosci 22, 1504-1529

Brauer J, Anwander A, Friederici AD (2011): Neuroanatomical prerequisites for language functions in the maturing brain. Cereb Cortex 21, 459-466

Braun AR, Varga M, Stager S, Schulz G, Selbie S, Maisog JM, Carson RE, Ludlow CL (1997): Altered patterns of cerebral activity during speech and language 
production in developmental stuttering. An $\mathrm{H} 2(15) \mathrm{O}$ positron emission tomography study. Brain $\underline{120}, 761-784$

Brown S, Ingham RJ, Ingham JC, Laird AR, Fox PT (2005): Stuttered and fluent speech production: An ALE meta-analysis of functional neuroimaging studies. Hum Brain Mapp 25, 105-117

Büchel C, Sommer M (2004): What causes stuttering? PLoS Biol 2, E46

Cai S, Tourville JA, Beal DS, Perkell JS, Guenther FH, Ghosh SS (2014): Diffusion imaging of cerebral white matter in persons who stutter: evidence for network-level anomalies. Front Hum Neurosci $8, \underline{54}$

Catani M, Jones DK, Dominic H (2005): Perisylvian language networks of the human brain. Ann Neurol 57, 8-16

Chang SE, Erickson KI, Ambrose NG, Hasegawa-Johnson MA, Ludlow CL (2008): Brain anatomy differences in childhood stuttering. Neurolmage 39, 13331344

Chang SE, Horwitz B, Ostuni J, Reynolds R, Ludlow CL (2011): Evidence of left inferior frontal-premotor structural and functional connectivity deficits in adults who stutter. Cereb Cortex 21, 2507-2518

Classen J, Liepert J, Wise SP, Hallett M, Cohen LG (1998): Rapid plasticity of human cortical movement representation induced by practice. $\mathrm{J}$ Neurophysiol $\underline{79}, 1117-1123$

Cykowski MD, Fox PT, Ingham RJ, Ingham JC, Robin DA (2010): A study of the reproducibility and etiology of diffusion anisotropy differences in developmental stuttering: A potential role for impaired myelination. Neurolmage 52, 1495-1504

Davis S, Howell P, Cooke F (2002): Sociodynamic relationships between children who stutter and their non-stuttering classmates. J Child Psychol Psychiatry 43, 939-948

Deecke L, Engel M, Lang W, Kornhuber HH (1986): Bereitschaftspotential preceding speech after holding breath. Exp Brain Res 65, 219-223

Di Lazzaro V, Oliviero A, Pilato F, Saturno E, Dileone M, Mazzone P, Insola A, Tonali P, Rothwell J (2004): The physiological basis of transcranial motor cortex stimulation in conscious humans. Clin Neurophysiol 115, 255-266

Eickhoff SB, Heim S, Zilles K, Amunts K (2009): A Systems Perspective on the Effective Connectivity of Overt Speech Production. Philos Trans Math Phys Eng Sci 367, 2399-2421

Fitch WT, Huber L, Bugnyar T (2010): Social cognition and the evolution of language: constructing cognitive phylogenies. Neuron 65 , 795-814

Fitzgerald PB, Fountain S, Daskalakis ZJ (2006): A comprehensive review of the effects of rTMS on motor cortical excitability and inhibition. Clin Neurophysiol 117, 2584-2596 
Floel A, Nagorsen U, Werhahn KJ, Ravindran S, Birbaumer N, Knecht S, Cohen LG (2004): Influence of somatosensory input on motor function in patients with chronic stroke. Ann Neurol 56, 206-212

Foundas AL, Bollich AM, Corey DM, Hurley M, Heilman KM (2001): Anomalous anatomy of speech-language areas in adults with persistent developmental stuttering. Neurology $\underline{57}, 207-215$

Fox PT, Ingham RJ, Ingham JC, Hirsch TB, Downs JH, Martin C, Jerabek P, Glass T, Lancaster JL (1996): A PET study of the neural systems of stuttering. Nature 382, 158-161

Friederici AD (2002): Towards a neural basis of auditory sentence processing. Trends Cogn Sci $\underline{6}, 78-84$

Friederici AD (2006): The brain differentiates human and non-human grammars: Functional localization and structural connectivity. Proc Natl Acad Sci USA 103, 2458-2463

Friederici AD (2011): The brain basis of language processing: from structure to function. Physiol Rev 91, 1357-1392

Friston KJ, Harrison L, Penny W (2003): Dynamic causal modelling. Neurolmage 19, 1273-1302

George MS, Aston-Jones G (2010): Noninvasive techniques for probing neurocircuitry and treating illness: vagus nerve stimulation (VNS), transcranial magnetic stimulation (TMS) and transcranial direct current stimulation (tDCS). Neuropsychopharmacology 35, 301-316

George MS, Wassermann EM, Williams WA, Callahan A, Ketter TA, Basser P, Hallett M, Post RM (1995): Daily repetitive transcranial magnetic stimulation (rTMS) improves mood in depression. NeuroReport 6 , 1853-1856

Geschwind N, Galaburda AM: Cerebral lateralization: biological mechanisms, associations, and pathology. MIT Press, Cambridge, Mass. 1985

Greenlee J, Oya H, Kawasaki H, Volkov IO, Kaufman OP, Kovach C, Howard MA, Brugge JF (2004): A functional connection between inferior frontal gyrus and orofacial motor cortex in human. J Neurophysiol 92, 1153-64

Grohnfeldt M: Redeflußstörung - mehr Fragen als Antworten? Zur Vielfalt des Erscheinungsbildes, vermuteten Bedingungsgefüges und therapeutischen Ansatzes. In: Grohnfeldt M (Hrsg.): Handbuch der Sprachtherapie. Bd. 5: Störung der Redefähigkeit. Ed. Marhold im wiss.-Verl. Spiess, Berlin 1992

Guenther FH (2006): Cortical interactions underlying the production of speech sounds. J Commun Disord 39, 350-365

Hagoort P, Levelt WJM (2009): The Speaking Brain. Science 326, 372-373

Harrington DL, Haaland $\mathrm{KY}$ : Sequencing and timing operations of the basal ganglia. In: Rosenbaum DA, Collyer CE (Hrsg.): Timing of behavior: neural, 
psychological, and computational perspectives. MIT Press, Cambridge, Mass. 1998

Hayes C: The ape in our house. Harper, New York 1951

Hickok G (2012): Computational neuroanatomy of speech production. Nat Rev Neurosci 13, 135-145

Hickok G, Poeppel D (2004): Dorsal and ventral streams: a framework for understanding aspects of the functional anatomy of language. Cognition 92 , 67-99

Hickok G, Poeppel D (2007): The cortical organization of speech processing. Nat Rev Neurosci 8, 393-402

Hummel FC, Cohen LG (2006): Non-invasive brain stimulation: a new strategy to improve neurorehabilitation after stroke? Lancet Neurol $\underline{5}, 708-712$

Jones RK (1966): Observations on stammering after localized cerebral injury. J Neurol Neurosurg Psychiatry 29, 192-195

Jueptner M, Weiller C (1998): A review of differences between basal ganglia and cerebellar control of movements as revealed by functional imaging studies. Brain J Neurol 121, 1437-1449

Jürgens U (2006): Die Evolution der Sprache: Vom Affenlaut zum Menschenwort. Biol Unserer Zeit 36, 362-368

Kaneko K, Kawai S, Fuchigami Y, Morita H, Ofuji A (1996): The effect of current direction induced by transcranial magnetic stimulation on the corticospinal excitability in human brain. Electroencephalogr Clin Neurophysiol 101, 478482

Keel JC, Smith MJ, Wassermann EM (2001): A safety screening questionnaire for transcranial magnetic stimulation. Clin Neurophysiol 112, 720

Klinke R, Pape H-C, Silbernagl S: Physiologie. Thieme, Stuttgart New York 2005

Korchounov A, Ilić TV, Ziemann U (2007): TMS-assisted neurophysiological profiling of the dopamine receptor agonist cabergoline in human motor cortex. J Neural Transm J Neural Transm 114, 223-229

Kornhuber HH, Deecke L (1965): Hirnpotentialänderungen bei Willkürbewegungen und passiven Bewegungen des menschen: Bereitschaftspotential und reafferente Potentiale. Pflugers Arch Physiol 284, 1-17

Kreisler A, Godefroy O, Delmaire C, Debachy B, Leclercq M, Pruvo JP, Leys D (2000): The anatomy of aphasia revisited. Neurology 54, 1117-1123

Lattermann C (2011): Frühkindliches Stottern: Abwarten oder sofort behandeln? Indikatoren für den Therapiebeginn auf der Basis von aktuellen. Forum Logopadie 25, 6-11 
Lefaucheur J-P, André-Obadia N, Antal A, Ayache SS, Baeken C, Benninger DH, Cantello R, Cincotta M, de Carvalho M, De Ridder D, et al. (2014): Evidence-based guidelines on the therapeutic use of repetitive transcranial magnetic stimulation (rTMS). Clin Neurophysiol 125, 2150-2206

Leon-Sarmiento FE, Paez E, Hallett M (2013): Nature and nurture in stuttering: a systematic review on the case of Moses. Neurol Sci 34, 231-237

Lichteim L (1885): On Aphasia. Brain ㄱ, 433-484

Lieberman P, McCarthy R (2007): Tracking the evolution of language and speechcomparing vocal tracts to identify speech capabilities. Expedition $\underline{49}, 15$

Lurija AR: Sprache und Bewusstsein. Volk und Wissen, Volkseigener Verlag, Berlin 1982

McAllister J, Collier J, Shepstone L (2012): The impact of adolescent stuttering on educational and employment outcomes: Evidence from a birth cohort study. J Fluen Disord 37, 106-121

Moore WHJ, Haynes WO (1980): Alpha hemispheric asymmetry and stuttering: some support for a segmentation dysfunction hypothesis. J Speech Hear Res 23, 229-247

Muellbacher W, Boroojerdi B, Ziemann U, Hallett M (2001): Analogous corticocortical inhibition and facilitation in ipsilateral and contralateral human motor cortex representations of the tongue. J Clin Neurophysiol 18 , 550-558

Natke U: Stottern: Erkenntnisse, Theorien, Behandlungsmethoden. Huber, Bern 2005

Neef NE, Jung K, Rothkegel H, Pollok B, von Gudenberg AW, Paulus W, Sommer $M$ (2011): Right-shift for non-speech motor processing in adults who stutter. Cortex J Devoted Study Nerv Syst Behav 47, 945-954

Neef NE, Hoang TNL, Neef A, Paulus W, Sommer M (2015): Speech dynamics are coded in the left motor cortex in fluent speakers but not in adults who stutter. Brain 138, 712-725

Nelson SF, Hunter N, Walter M (1945): Stuttering in twin types. J Speech Disord 10, 335-343

Neumann K, Preibisch C, Euler HA, von Gudenberg AW, Lanfermann H, Gall V, Giraud A-L (2005): Cortical plasticity associated with stuttering therapy. J Fluen Disord 30, 23-39

Oldfield RC (1971): The assessment and analysis of handedness: the Edinburgh inventory. Neuropsychologia $\underline{9}, 97-113$

Orton ST (1927): Studies in Stuttering; Introduction. Arch Neurol Psychiatry 18, 671-672 
Orton ST (1928): A Physiological Theory of Reading Disability and Stuttering in Children. N Engl J Med 199, 1046-1052

Paulus W (2009): International conference on transcranial magnetic and direct current stimulation. Dtsch Arzteblatt Int 106, 143-144

Peeva MG, Guenther FH, Tourville JA, Nieto-Castanon A, Anton J-L, Nazarian B, Alario F-X (2010): Distinct representations of phonemes, syllables, and supra-syllabic sequences in the speech production network. Neurolmage $\underline{50}, 626-638$

Primaßin A, Olthoff A, Sommer M (2017): „Das habe ich jetzt nicht verstanden ...“ - Sprach- oder Sprechstörung? InFo Neurol Psychiatr 19, 32-43

Prins D: Theories of stuttering as event and disorder: implications for speech production processes. In: Peters HFM, Hulstijin W, Starkweather CW (Hrsg.): Speech motor control and stuttering. Elsevier Science, Amsterdam: 1991

Rauschecker JP, Scott SK (2009): Maps and streams in the auditory cortex: nonhuman primates illuminate human speech processing. Nat Neurosci 12 , 718-724

Riley GD: Stuttering severity instrument for children and adults. 3. Auflage; ProEd, Austin, TX 1994

Ringo CC, Dietrich S (1995): Neurogenic stuttering: An analysis and critique. J Med Speech Lang Pathol $\underline{3}, 111-122$

Rödel MR, Laskawi R, Markus H (2003): Tongue representation in the lateral cortical motor region of the human brain as assessed by transcranial magnetic stimulation. Ann Otol Rhinol Laryngol 112, 71-76

Rösler K., Hess C., Heckmann R, Ludin H. (1989): Significance of shape and size of the stimulating coil in magnetic stimulation of the human motor cortex. Neurosci Lett Neurosci Lett 100, 347-352

Rossi S, Hallett M, Rossini PM, Pascual-Leone (2009): Safety, ethical considerations, and application guidelines for the use of transcranial magnetic stimulation in clinical practice and research. Clin Neurophysiol 120, 2008-2039

Rothwell JC (1997): Techniques and mechanisms of action of transcranial stimulation of the human motor cortex. J Neurosci Methods $\underline{74}, 113-122$

Salmelin R, Schnitzler A, Schmitz F, Freund H-J (2000): Single word reading in developmental stutterers and fluent speakers. Brain J Neurol 123, 11841202

Sandrieser P, Schneider P: Stottern im Kindesalter. 2. Auflage; Thieme, Stuttgart 2003

Sandrieser P, Schneider P: Stottern im Kindesalter. 3. Auflage; Thieme, Stuttgart 2008 
Schwegler JS, Lucius R: Der Mensch: Anatomie und Physiologie. Georg Thieme Verlag, Stuttgart New York 2016

Seemann M: Die Bedeutung der Zwillingspathologie für die Erforschung von Sprachleiden. Band Bd. I; Arch. f. Sprach- und Stimmheilkunde u. angew. Phonetik 1937

Siebner HR, Ziemann U: Das TMS-Buch: Handbuch der transkraniellen Magnetstimulation. Springer Medizin, Heidelberg 2007

Sommer M (2002): Disconnection of speech-relevant brain areas in persistent developmental stuttering. Lancet 360, 380-383

Sommer M, Classen J, Cohen LG, Hallett M (2001): Time course of determination of movement direction in the reaction time task in humans. $J$ Neurophysiol 86, 1195-1201

St Clare T, Menzies R, Onslow M, Packman A, Thompson R, Block S (2009): Unhelpful thoughts and beliefs linked to social anxiety in stuttering: development of a measure. Int J Lang Commun Disord 44, 338-351

Svensson P, Romaniello A, Wang K, Arendt-Nielsen L, Sessle BJ (2006): One hour of tongue-task training is associated with plasticity in corticomotor control of the human tongue musculature. Exp Brain Res 173, 165-173

Tergau F, Sommer M, Nitsche MA, Paulus W (2001): Transkranielle Kortexstimulation beim Menschen. E-Neuroforum 7

Terumitsu M, Fujii Y, Suzuki K, Kwee IL, Nakada T (2006): Human primary motor cortex shows hemispheric specialization for speech. NeuroReport 17, 1091-1095

Tran Y, Blumgart E, Craig A (2011): Subjective distress associated with chronic stuttering. J Fluen Disord 36, 17-26

Travis LE (1978): The cerebral dominance theory of stuttering: 1931-1978. J Speech Hear Disord 43, 278-281

Trepel M: Neuroanatomie: Struktur und Funktion; 3., neu bearb. Aufl., 2. Nachdruck; Urban \& Fischer, München 2005

Ueno S, Tashiro T, Harada K (1988): Localized stimulation of neural tissues in the brain by means of a paired configuration of time-varying magnetic fields. $\mathrm{J}$ Appl Phys 64, 5862-5864

Van Riper C: The Nature of Stuttering. 2. Auflage; Prentice-Hall, Englewood Cliffs 1982

Vigneau M, Beaucousin V, Hervé PY, Duffau H, Crivello F, Houdé O, Mazoyer B, Tzourio-Mazoyer N (2006): Meta-analyzing left hemisphere language areas: Phonology, semantics, and sentence processing. Neurolmage 30, 14141432 
Walla P, Mayer D, Deecke L, Thurner S (2004): The lack of focused anticipation of verbal information in stutterers: a magnetoencephalographic study. Neurolmage 22, 1321-1327

Walle EL (1971): Intracarotid sodium amytal testing on normal, chronic adult stutterers. J Speech Hear Disord 36, 561

Walter WG (1964): Slow potential waves in the human brain associated with expectancy, attention and decision. Arch Psychiatr Nervenkr 206, 309-322

Wassermann E (1996): Use and safety of a new repetitive transcranial magnetic stimulator. Electroencephalogr Clin Neurophysiol Mot Control 101, 412-417

Wassermann EM (1998): Risk and safety of repetitive transcranial magnetic stimulation: report and suggested guidelines from the International Workshop on the Safety of Repetitive Transcranial Magnetic Stimulation, June 5-7, 1996. Electroencephalogr Clin Neurophysiol 108, 1-16

Watkins KE, Paus T, Lerch JP, Zijdenbos A, Collins DL, Neelin P, Taylor J, Worsley KJ, Evans AC (2001): Structural Asymmetries in the Human Brain: a Voxel-based Statistical Analysis of 142 MRI Scans. Cereb Cortex 11, 868-877

Watkins KE, Smith SM, Davis S, Howell P (2008): Structural and functional abnormalities of the motor system in developmental stuttering. Brain 131, 50-59

Weber-Fox C, Usler E (2015): Neurodevelopment for syntactic processing distinguishes childhood stuttering recovery versus persistence. J Neurodev Disord $\underline{7}, 1-22$

West R, Nelson S, Berry M (1939): The heredity of stuttering. Q J Speech 25, 2330

Wingate, M. (1964): A Standard Definition of Stuttering. J Speech Hear Disord 29, 484-9

Wingate ME: Foundations of stuttering. Academic Press, San Diego 2002

Wu JC, Maguire G, Riley G, Lee A, Keator D, Tang C, Fallon J, Najafi A (1997): Increased dopamine activity associated with stuttering. NeuroReport NeuroReport $\underline{8}, 767-770$

Yairi E (1983): The onset of stuttering in two- and three-year-old children: a preliminary report. J Speech Hear Disord $\underline{48}$, 171-177

Yairi E: Disfluency characteristics of childhood stuttering. In: R.F. Curlee \& G.M. Siegel (Hrsg.), Nature and Treatment of Stuttering: New Directions. 2. Auflage; Allyn and Bacon, Boston 1997

Yairi E, Ambrose N (1992): Onset of Stuttering in Preschool Children. J Speech Lang Hear Res $\underline{35}, 782-788$ 
Ziemann U, Tergau F, Bruns D, Baudewig J, Paulus W (1997): Changes in human motor cortex excitability induced by dopaminergic and anti-dopaminergic drugs. EEM Electroencephalogr Clin Neurophysiol Electromyogr Mot Control 105, 430-437

Open Textbook Library. https://open.umn.edu/opentextbooks/BookDetail.aspx? bookld= 48; Zugriff am 13.12.2017 


\section{Danksagung}

Bei Herrn Prof. Dr. med. W. Paulus, dem Direktor der Abteilung für Klinische Neurophysiologie der Georg-August-Universität Göttingen, möchte ich mich dafür bedanken, die Möglichkeit zur Anfertigung der vorliegenden Promotionsarbeit erhalten zu haben.

Herrn Prof. Dr. med. M. Sommer danke ich insbesondere für die Überlassung des Themas und die Betreuung dieser Arbeit.

Frau Dr. ret. nat. N. Neef möchte ich danken für die Einführung in die Methodik der TMS sowie für die Unterstützung und Betreuung der gesamten Phase der Datenerhebung und Auswertung. Ebenfalls bedanke ich mich für die Unterstützung bei der Verwirklichung und Publikation der Arbeit.

Bei Herrn G. Ambrus bedanke ich mich für die technischen Unterstützung bei der Durchführung der Untersuchung.

Für die Unterstützung bei der Erhebung der Daten der Probanden mit Stottern bedanke ich mich ganz herzlich bei Herrn Dr. A. Wolff von Gudenberg vom Institut der Kasseler Stottertherapie sowie bei der Stotterer-Selbsthilfegruppe in Göttingen.

Weiterhin möchte ich mich bei allen Probanden bedanken, die sich freundlicherweise für die Experimente zur Verfügung gestellt haben.

Schließlich gilt mein Dank allen kritisch beratenden Freunden und Kollegen, hier insbesondere Herrn Dr. med. S. Schade, Frau Dr. med. D. Geisenberger, Frau A. Ebert, Frau Dr. K. Loscha, Herrn Prof. Dr. L. Suwala, Herrn H. Hoang, Frau D. Nguyen. 